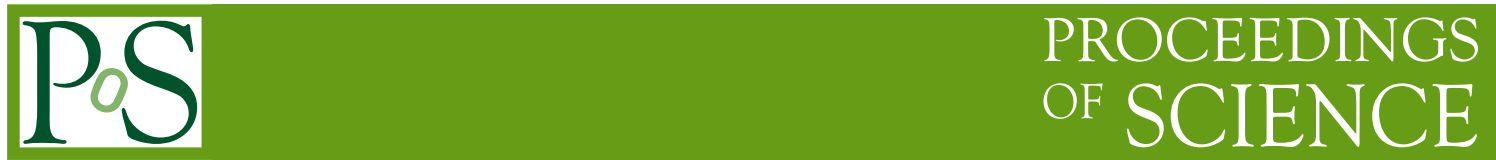

\title{
Rotational Submanifolds in Pseudo-Euclidean Spaces
}

\section{Bruno Mendonça Rey dos Santos*}

State University of Londrina, Department of Mathematics

E-mail: brunomrs@uel.br

We define rotational submanifolds in pseudo-euclidean spaces $\mathbb{R}_{t}^{n}$. We use the rotational immersion to classify all rotational submanifolds of $\mathbb{L}^{n}$ and we also generalize a result showing sufficient conditions for a riemannian submanifold of $\mathbb{R}_{t}^{n}$ be a rotational submanifold.

$3 d$ International Satellite Conference on Mathematical Methods in Physics 21 - 26 October, 2013

Londrina - PR (Brazil)

\footnotetext{
* Speaker.
} 


\section{Introduction}

Rotational submanifolds play an important role at submanifolds theory of riemannian manifolds (see, for example, [1] and [2]). They also play an important role at the study of marginally trapped surfaces which, by their turn, are important to study black roles (see [3] and [4]).

There are lots of definitions of rotational submanifolds: rotational submanifolds in $\mathbb{R}^{n}$ (see [5]), rotational hypersurfaces in constant curvature spaces (see [1]), rotational hypersurfaces in $\mathbb{S}^{n} \times \mathbb{R}$ and $\mathbb{H}^{n} \times \mathbb{R}$ in (see [6]), and other definitions. But constant curvature spaces, $\mathbb{S}^{n} \times \mathbb{R}$ and $\mathbb{H}^{n} \times \mathbb{R}$ are submanifolds of pseudo-euclidean spaces, therefore, it is possible to use one definition which will serve at all theses cases, we just have to define rotational submanifolds in pseudoeuclidean spaces.

In order to define rotational submanifolds in pseudo-euclidean spaces, some notations are used. A pseudo-euclidean space $\mathbb{R}_{t}^{n}, t \leq n$, is the vector space $\mathbb{R}^{n}$ together with the inner product given by

$$
\langle x, y\rangle:=-\sum_{i=1}^{t} x_{i} y_{i}+\sum_{i=t+1}^{n} x_{i} y_{i}
$$

where $x=\left(x_{1}, \cdots, x_{n}\right), y=\left(y_{1}, \cdots, y_{n}\right)$ and the symbol ":=" means "equal by definition". We are going to use the following definitions:

$$
\begin{aligned}
& \|x\|^{2}:=\langle x, x\rangle ; \\
& \mathbb{S}^{n}:=\left\{x \in \mathbb{R}^{n} \mid\|x\|^{2}=1\right\} ; \\
& \mathbb{S}^{n}(p, r):=\left\{x \in \mathbb{R}_{t}^{n} \mid\|x-p\|^{2}=r^{2}\right\} ; \\
& \mathbb{S}^{n}(p,-r):=\left\{x \in \mathbb{R}_{t}^{n} \mid\|x-p\|^{2}=-r^{2}\right\} ; \\
& \mathbb{H}^{n}:=\left\{x \in \mathbb{S}^{n}(0,-1) \mid x_{1}>0\right\} ; \\
& \mathscr{L}:=\left\{x \in \mathbb{R}_{t}^{n} \mid\|x\|^{2}=0\right\}, \text { is the light cone; } \\
& \mathscr{L}^{*}:=\left\{x \in \mathbb{R}_{t}^{n} \mid\|x\|^{2}=0 \text { and } x \neq 0\right\}, \text { is the light cone without the origin. }
\end{aligned}
$$

Let $x \in \mathbb{R}_{t}^{n}$. We say that $x$ is: spacelike, if $\|x\|^{2}>0$; timelike, if $\|x\|^{2}<0$; or lightlike, if $\|x\|^{2}=0$. Given $V \subset \mathbb{R}_{t}^{n}$ a vector subspace, we say that $V$ is:

- spacelike, if every vector of $V$ is spacelike;

- timelike, if there is a basis of $V$ in which the inner product of two vectors of $V$ can be written like

$$
\langle v, w\rangle=-\sum_{i=1}^{s} v_{i} w_{i}+\sum_{i=s+1}^{m} v_{i} w_{i}
$$

where $s \leq t$ and $m \leq n$;

- lightlike, if the inner product in $V$ is degenerated.

Let $\mathbb{R}^{n-q-1}$ a vector subspace of $\mathbb{R}_{t}^{n}$, with $1 \leq q \leq n-2$. Lets denote the group of all linear isometries of $\mathbb{R}_{t}^{n}$ by $\mathrm{O}_{t}(n)$ and by $\mathrm{O}(q+1)$ the subgroup of $\mathrm{O}_{t}(n)$ which fixes every point of $\mathbb{R}^{n-q-1}$.

Definition 1. Let $\mathbb{R}^{n-q}$ be a vector subspace of $\mathbb{R}_{t}^{n}$ and $f: N^{m-q} \rightarrow \mathbb{R}^{n-q}$ be an immersion such that $\mathbb{R}^{n-q-1} \subset \mathbb{R}^{n-q}$ and $f(N) \cap \mathbb{R}^{n-q-1}=\varnothing$. The rotational submanifold with axis $\mathbb{R}^{n-q-1}$ on $f$ is the union of the orbits of points of $f(N)$ under the action of the group $\mathrm{O}(q+1)$, ie., it is the set

$$
\{A(f(x)) \mid x \in N \text { and } A \in \mathrm{O}(q+1)\} \text {. }
$$


In the euclidean case $\left(\mathbb{R}_{t}^{n}=\mathbb{R}^{n}\right)$, the above definition is the same given in [5]. A more general definition for the euclidean case can be found in [7].

Our first objective is to prove the following proposition:

Proposition 2. Let $\mathbb{R}^{n-q-1} \subset \mathbb{R}^{n-q}$ be two vector subspaces of $\mathbb{R}_{t}^{n}$ and $f: N^{m-q} \rightarrow \mathbb{R}^{n-q}$ an immersion such that $f(N) \cap \mathbb{R}^{n-q-1}=\varnothing$. Let also $M$ be a rotational submanifold on $f$, with axis $\mathbb{R}^{n-q-1}$.

1. Lets suppose that $\mathbb{R}^{n-q-1}$ has index $s$ (ie. $\mathbb{R}^{n-q-1}=\mathbb{R}_{s}^{n-q-1}$ ), $\mathbb{R}_{t-s}^{q+1}:=\left(\mathbb{R}_{s}^{n-q-1}\right)^{\perp}$ and $\pi: \mathbb{R}_{t}^{n} \rightarrow \mathbb{R}_{s}^{n-q-1}$ is the orthogonal projection of $\mathbb{R}_{t}^{n}=\mathbb{R}_{t-s}^{q+1} \oplus \mathbb{R}_{s}^{n-q-1}$ on $\mathbb{R}_{s}^{n-q-1}$.

(a) If $\mathbb{R}^{n-q}$ has index $s\left(\mathbb{R}^{n-q}=\mathbb{R}_{s}^{n-q}\right)$, lets consider $\mathbb{S}(0,1) \subset \mathbb{R}_{t-s}^{q+1}$ and $X_{1} \in \mathbb{R}_{s}^{n-q} \cap$ $\left(\mathbb{R}_{s}^{n-q-1}\right)^{\perp}$ a unit spacelike vector. In this case, we can define $\bar{M}$ and $g: N \times \mathbb{S}(0,1) \rightarrow$ $\bar{M}$ by

$\bar{M}:=\left\{f_{1}(x) \xi+\pi(f(x)) \mid x \in N\right.$ and $\left.\xi \in \mathbb{S}(0,1)\right\} \quad$ and $\quad g(x, \xi):=f_{1}(x) \xi+\pi(f(x))$, where $f_{1}(x):=\left\langle f(x), X_{1}\right\rangle$.

(b) If $\mathbb{R}^{n-q}=\mathbb{R}_{s+1}^{n-q}$, lets consider $\mathbb{S}(0,-1) \subset \mathbb{R}_{t-s}^{q+1}$ and $X_{1} \in \mathbb{R}_{s+1}^{n-q} \cap\left(\mathbb{R}_{s}^{n-q-1}\right)^{\perp}$ a unit timelike vector. In this case, we can define $\bar{M}$ and $g: N \times \mathbb{S}(0,-1) \rightarrow \bar{M}$ by

$\bar{M}:=\left\{f_{1}(x) \xi+\pi(f(x)) \mid x \in N\right.$ and $\left.\xi \in \mathbb{S}(0,-1)\right\} \quad$ and $\quad g(x, \xi):=f_{1}(x) \xi+\pi(f(x))$,

where $f_{1}(x):=-\left\langle f(x), X_{1}\right\rangle$.

(c) If $\mathbb{R}^{n-q}$ is lightlike, lets consider $\mathscr{L}^{*} \subset \mathbb{R}_{t-s}^{q+1}$ and $X_{1} \in \mathbb{R}^{n-q} \cap\left(\mathbb{R}_{s}^{n-q-1}\right)^{\perp}$ a lightlike vector. In this case, we can define $\bar{M}$ and $g: N \times \mathscr{L}^{*} \rightarrow \bar{M}$ by

$$
\bar{M}:=\left\{f_{1}(x) \xi+\pi(f(x)) \mid x \in N \text { and } \xi \in \mathscr{L}^{*}\right\} \quad \text { and } \quad g(x, \xi):=f_{1}(x) \xi+\pi(f(x)),
$$

where $f_{1}(x)$ is the component of $f(x)$ in the $X_{1}$ direction, ie., $f(x)=f_{1}(x) X_{1}+\pi(f(X))$.

2. Let suppose that $\mathbb{R}^{n-q-1}$ is lightlike and there are non-degenerated vector subspaces $U, V \subset$ $\mathbb{R}_{t}^{n}$ and lightlike vectors $X_{1}$ and $X_{2}$ such that $\left\langle X_{1}, X_{2}\right\rangle=1, \mathbb{R}^{n-q-1}=\operatorname{span}\left\{X_{2}\right\} \oplus U$ and $\mathbb{R}_{t}^{n}=\operatorname{span}\left\{X_{1}, X_{2}\right\} \oplus U \oplus V$. In this case, let $\pi: \operatorname{span}\left\{X_{1}\right\} \oplus V \oplus \mathbb{R}^{n-q-1} \rightarrow \mathbb{R}^{n-q-1}$ be the projection application.

(a) If $\mathbb{R}^{n-q}=\operatorname{span}\left\{X_{1}, X_{2}\right\} \oplus U$, lets define $\bar{M}$ and $g: N \times V \rightarrow \mathbb{R}_{t}^{n}$ by

$$
\begin{aligned}
& \bar{M}:=\left\{f_{1}(x)\left(X_{1}+v-\frac{\|v\|^{2}}{2} X_{2}\right)+\pi(f(x)) \mid x \in N \text { and } v \in V\right\} \quad \text { and } \\
& g(x, v):=f_{1}(x)\left(X_{1}+v-\frac{\|v\|^{2}}{2} X_{2}\right)+\pi(f(x))
\end{aligned}
$$

where $f_{1}(x)=\left\langle f(x), X_{2}\right\rangle$. 
(b) If $\mathbb{R}^{n-q}=\operatorname{span}\left\{w, X_{2}\right\} \oplus U$, where $w \in V$ is a unit vector, lets consider $\varepsilon:=\|w\|^{2}$ and $\mathbb{S}(0, \varepsilon) \subset V$ and we can define $\bar{M}$ and $g: N \times \mathbb{S}(0, \varepsilon) \times \mathbb{R} \rightarrow \mathbb{R}_{t}^{n}$ by

$$
\begin{aligned}
& \bar{M}:=\left\{f_{1}(x)\left(\lambda X_{2}+\xi\right)+\pi(f(x)) \mid x \in N, \xi \in \mathbb{S}(0, \varepsilon) \text { and } \lambda \in \mathbb{R}\right\} \quad \text { and } \\
& g(x, \xi, 1):=f_{1}(x)\left(\lambda X_{2}+\xi\right)+\pi(f(x)),
\end{aligned}
$$

where $f_{1}(x)=\varepsilon\langle f(x), w\rangle$.

In any of the above cases, $M=\bar{M}$. Furthermore, in the cases (I.1), (I.2) and (II.1), $g$ is an immersion. With the hypothesis that $N$ is a riemannian manifold and $f$ is an isometric immersion, $g$ is also an immersion in the cases (I.3) and (II.2).

This proposition studies some of the possible cases for rotational submanifolds in $\mathbb{R}_{t}^{n}$, but there are some other cases which were not studied, for example, the case in which $\mathbb{R}^{n-q-1}=$ $\mathbb{R}_{s}^{n-q-1-\ell} \oplus \operatorname{span}\left\{v_{1}, \cdots, v_{\ell}\right\}$, where $v_{1}, \cdots, v_{\ell}$ are orthogonal lightlike vectors. Besides that, if $t=1$, that is, $\mathbb{R}_{t}^{n}=\mathbb{L}^{n}$ is the Lorentz space, then Proposition 2 is enough.

Corollary 3. Proposition 2 classifies all rotational submanifolds in $\mathbb{L}^{n}$ on an immersion $f$, according to the codomain of $f$ and to the rotational axis.

Once we have proved those results, we want to show another one but, first, we need some definitions.

Let $M_{s}^{m}$ and $N_{t}^{n}$ be two pseudo-riemannian manifolds and $f: M_{s}^{m} \rightarrow N_{t}^{n}$ an isometric immersion. Given a vector $\eta \in T_{x}^{\perp} M$, it's conformal nullity subspace is given by

$$
E_{\eta}(x):=\left\{X \in T_{x} M \mid \alpha(X, Y)=\langle X, Y\rangle \eta, \forall Y \in T_{x} M\right\} .
$$

We say that $\eta \in \Gamma\left(T^{\perp} M\right)$ is a principal normal if $\operatorname{dim} E_{\eta}(x) \geq 1$, for all $x \in M$. If $\eta$ is a principal normal, $E_{\eta}$ has constant dimension and $\eta$ is parallel in the normal connection of $f$ along $E_{\eta}$, then $\eta$ is called a Dupin normal of $f$. In this case, the number $\operatorname{dim} E_{\eta}$ is the multiplicity of $\eta$.

A distribution $\mathscr{D}$ in a riemannian manifold $M^{n}$ is umbilical if there exists a vector field $\varphi \in \Gamma\left(\mathscr{D}^{\perp}\right)$ such that $\nabla_{X}^{h} Y=\langle X, Y\rangle \varphi$, for all $X$ and all $Y$ in $\Gamma(\mathscr{D})$, where $\nabla_{X}^{h} Y$ is the orthogonal projection of $\nabla_{X} Y$ on $\mathscr{D}^{\perp}$. The vector $\varphi$ is called mean curvature vector of the umbilical distribution $\mathscr{D}$. If $\mathscr{D}$ is umbilical and it's mean curvature vector is null $(\varphi \equiv 0)$, then $\mathscr{D}$ is called totally geodesic. $\mathscr{D}$ is called spherical if $\mathscr{D}$ is umbilical and $\nabla_{X}^{h} \varphi=0$, for every $X \in \Gamma(\mathscr{D})$.

Our main result is the following theorem, which generalizes a similar theorem made in [5] for the euclidean case:

Theorem 1. Let $M^{m}$ be a riemannian manifold, $f: M^{m} \rightarrow \mathbb{R}_{t}^{n}$ an isometric immersion and $\eta$ a Dupin normal of $f$ with multiplicity $q$ and such that $\eta \neq 0$ in every point of $M$. If $E_{\eta}^{\perp}$ is totally geodesic, then there exists a rotational immersion $g$ such that $f(M)$ is a subset of the image of $g$. Furthermore, we have one of the following cases:

1. There is an orthogonal decomposition $\mathbb{R}_{t}^{n}=\mathbb{R}^{q+1} \ominus \mathbb{R}_{t}^{m-q-1}$ such that $g: N^{m-q} \times \mathbb{S}^{q} \rightarrow$ $\mathbb{R}^{q+1} \oplus \mathbb{R}_{t}^{n-q-1}$ is given by

$$
g(x, y)=p+r(x) y+h(x),
$$

where $p \in \mathbb{R}_{t}^{n}$ is a fixed point, $r(x)>0, r(x) y \in \mathbb{R}^{q+1}, h(x) \in \mathbb{R}_{t}^{n-q-1}$ and $\mathbb{R}_{t}^{n-q-1}$ is the rotational axis. 
2. There is an orthogonal decomposition $\mathbb{R}_{t}^{n}=\mathbb{L}^{q+1} \ominus \mathbb{R}_{t-1}^{n-q-1}$ such that $g: N^{m-q} \times \mathbb{S}(0,-1) \rightarrow$ $\mathbb{L}^{q+1} \oplus \mathbb{R}_{t-1}^{n-q-1}$ is given by

$$
g(x, y)=p+r(x) y+h(x),
$$

where $p \in \mathbb{R}_{t}^{n}$ is a fixed point, $\mathbb{S}(0,-1) \subset \mathbb{L}^{q+1}, r(x)>0, r(x) y \in \mathbb{L}^{q+1}, h(x) \in \mathbb{R}_{t-1}^{n-q-1}$ and $\mathbb{R}_{t-1}^{n-q-1}$ is the rotational axis.

3. There are lightlike vectors $e_{1}, e_{2} \in \mathbb{R}_{t}^{n}$ and an orthogonal decomposition $\mathbb{R}_{t}^{n}=\operatorname{span}\left\{e_{1}, e_{2}\right\} \oplus$ $\mathbb{R}^{q} \oplus \mathbb{R}_{t-2}^{n-q-2}$ such that $\left\langle e_{1}, e_{2}\right\rangle=1$ and $g: N^{m-q} \times \mathbb{R}^{q} \rightarrow \mathbb{R}_{t}^{n}$ is given by

$$
g(x, y)=q+g_{1}(x) e_{1}+\left[g_{2}(x)-g_{1}(x) \frac{\|y\|^{2}}{2}\right] e_{2}+g_{1}(x) y+g_{3}(x)
$$

where $q \in \mathbb{R}_{t}^{n}$ is a fixed point, $g_{1}(x)>0, g_{3}(x) \in \mathbb{R}_{t-s-2}^{n-q-2}$ and $\operatorname{span}\left\{e_{2}\right\} \oplus \mathbb{R}_{t-2}^{n-q-2}$ is the rotational axis.

In [9], this theorem is used to to show that some umbilical submanifolds of a product of two constat curvature spaces are rotational submanifols in $\mathbb{R}_{t}^{N}$.

\section{Proof of Proposition 2 and Corollary 3}

Let $\mathscr{L}^{*}$ be the light cone without the null vector (origin).

Proof of the cases (I) of the Proposition 2.

Let $M:=\{A(f(x)) \mid x \in N$ and $A \in \mathrm{O}(q+1)\}$ be a rotational submanifold on $f$. We have to show that $M=\bar{M}$ and that $g$ is an immersion.

(I.1): Let $\mathbb{R}^{n-q}=\mathbb{R}_{s}^{n-q}$. Since $\mathbb{R}_{s}^{n-q-1}$ is a vector subspace of $\mathbb{R}_{s}^{n-q}$, there exists a unit spacelike vector $X_{1} \in \mathbb{R}_{s}^{n-q} \cap\left(\mathbb{R}_{s}^{n-q-1}\right)^{\perp}$. Thus, $f(x)=f_{1}(x) X_{1}+\pi(f(x))$, where $f_{1}(x):=\left\langle f(x), X_{1}\right\rangle$.

Affirmation 1: $M \subset \bar{M}$.

If $A \in \mathrm{O}(q+1)$, then

$$
A(f(x))=A\left(f_{1}(x) X_{1}+\pi(f(x))=f_{1}(x) A\left(X_{1}\right)+A(\pi(f(x))) .\right.
$$

But,

$$
A(\pi(f(x)))=\pi(f(x)) \quad \text { and } \quad\left\langle A\left(X_{1}\right), Y\right\rangle=\left\langle A\left(X_{1}\right), A(Y)\right\rangle=\left\langle X_{1}, Y\right\rangle=0,
$$

for all $Y \in \mathbb{R}_{s}^{n-q-1}$, because $A$ fixes the points of $\mathbb{R}_{s}^{n-q-1}$.

Thus $A\left(X_{1}\right) \in \mathbb{S}(0,1) \subset \mathbb{R}_{n-s}^{q+1} \perp \mathbb{R}_{s}^{n-q-1}$, since $A\left(X_{1}\right) \perp \mathbb{R}_{s}^{n-q-1}$ and $\left\|A\left(X_{1}\right)\right\|^{2}=\left\|X_{1}\right\|^{2}=1$. Therefore $A(f(x))=f_{1}(x) A\left(X_{1}\right)+\pi(f(x)) \in\left\{f_{1}(x) \xi+\pi(f(x)) \mid x \in N\right.$ and $\left.\xi \in \mathbb{S}(0,1)\right\}$. $\checkmark$

Affirmation 2: $\bar{M} \subset M$.

Let $x \in N$ and $\xi \in \mathbb{S}(0,1) \subset \mathbb{R}_{t-s}^{q+1} \perp \mathbb{R}_{s}^{n-q-1}$. Lets assume that $\left\{X_{1}, X_{2}, \cdots, X_{q+1}\right\}$ and $\left\{\xi, Y_{2}, \cdots, Y_{q+1}\right\}$ are two orthonormal basis of $\mathbb{R}_{t-s}^{q+1}$ such that $\left\|X_{i}\right\|^{2}=\left\|Y_{i}\right\|^{2}$. If $\left\{X_{q+2}, \cdots, X_{n}\right\}$ is an orthonormal basis of $\mathbb{R}_{s}^{n-q-1}$, then we can define $A \in \mathrm{O}_{t}(n)$ by

$$
A\left(X_{i}\right)= \begin{cases}\xi, & \text { if } i=1 \\ Y_{i}, & \text { if } i=2, \cdots, q+1 \\ X_{i}, & \text { if } i=q+2, \cdots, n\end{cases}
$$


It is clear that $A \in \mathrm{O}(q+1)$ and $f_{1}(x) \xi+\pi(f(x))=f_{1}(x) A\left(X_{1}\right)+A(\pi(f(x)))=A(f(x)) . \checkmark$

Affirmation 3: $g$ is an immersion.

In deed, calculating $\operatorname{dg}(x, \xi)\left(v_{1}, v_{2}\right)$ we get

$$
\mathrm{d} g(x, \xi)\left(v_{1}, v_{2}\right)=\left\langle\mathrm{d} f(x) v_{1}, X_{1}\right\rangle \xi+\left\langle f(x), X_{1}\right\rangle v_{2}+\pi\left(\mathrm{d} f(x) v_{1}\right) .
$$

If $\mathrm{d} g(x, \xi)\left(v_{1}, v_{2}\right)=0$, then $\left\langle\mathrm{d} f(x) v_{1}, X_{1}\right\rangle \xi=0,\left\langle f(x), X_{1}\right\rangle v_{2}=0$ and $\pi\left(\mathrm{d} f(x) v_{1}\right)=0$, since $v_{2} \perp \xi$ and $\xi, v_{2} \in \mathbb{R}_{t-s}^{q+1} \perp \mathbb{R}_{s}^{n-q-1}$. Thus

$$
\begin{cases}\left\langle\mathrm{d} f(x) v_{1}, X_{1}\right\rangle=0, & \text { cause } \xi \neq 0 ; \\ v_{2}=0, & \text { cause } f(x) \notin \mathbb{R}_{s}^{n-q-1}, \text { ie. },\left\langle f(x), X_{1}\right\rangle \neq 0 ; \text { and } \\ \pi\left(\mathrm{d} f(x) v_{1}\right)=0 . & \end{cases}
$$

Thus

$$
\left\langle\mathrm{d} f(x) v_{1}, X_{1}\right\rangle X_{1}+\pi\left(\mathrm{d} f(x) v_{1}\right)=\mathrm{d} f(x) v_{1}=0 \Rightarrow\left(v_{1}, v_{2}\right)=(0,0) .
$$

Therefore $g$ is an immersion. $\checkmark$ •

(I.2): The proof is analogous to the proof of the previous case. •

(I.3): Lets assume that $\mathbb{R}^{n-q}$ is lightlike (nondegenerate). Since $\mathbb{R}_{s}^{n-q-1}$ is a vector subspace of $\mathbb{R}^{n-q}$, there exists a lightlike vector $X_{1} \in \mathbb{R}^{n-q} \cap\left(\mathbb{R}_{s}^{n-q-1}\right)^{\perp}$. Thus, $f(x)=f_{1}(x) X_{1}+\pi(f(x))$.

Affirmation 1: $M \subset \bar{M}$.

Analogous to the Affirmation 1 of the case (I.1). $\checkmark$

Affirmation 2: $\bar{M} \subset M$.

Let $x \in N$ and $\xi \in \mathscr{L}^{*} \subset \mathbb{R}_{t-s}^{q+1}=\left(\mathbb{R}_{s}^{n-q-1}\right)^{\perp}$ and lets consider $\left\{X_{1}, X_{2}, \cdots, X_{q+1}\right\}$ and $\left\{\xi, Y_{2}, \cdots, Y_{q+1}\right\}$ two basis of $\mathbb{R}_{t-s}^{q+1}$ such that

- $X_{1}, X_{2}, \xi$ and $Y_{2}$ are lightlike;

- $\left\langle X_{1}, X_{2}\right\rangle=1=\left\langle\xi, Y_{2}\right\rangle$;

- $\left\{X_{3}, \cdots, X_{q+1}\right\}$ and $\left\{Y_{3}, \cdots, Y_{q+1}\right\}$ are orthonormal sets;

- $\left\{X_{1}, X_{2}\right\} \perp\left\{X_{3}, \cdots, X_{q+1}\right\}$ and $\left\{\xi, Y_{2}\right\} \perp\left\{Y_{3}, \cdots, Y_{q+1}\right\}$.

If $\left\{X_{q+2}, \cdots, X_{n}\right\}$ is an orthonormal basis of $\mathbb{R}_{s}^{n-q-1}$, then we can define $A \in \mathrm{O}_{t}(n)$ by

$$
A\left(X_{i}\right)= \begin{cases}\xi, & \text { if } i=1 \\ Y_{i}, & \text { if } i \in\{2, \cdots, q+1\} \\ X_{i}, & \text { if } i \in\{q+2, \cdots, n\}\end{cases}
$$

Thus, $A \in \mathrm{O}(q+1)$ and $f_{1}(x) \xi+\pi(f(x))=f_{1}(x) A\left(X_{1}\right)+A(\pi(f(x)))=A(f(x)) \cdot \checkmark$

Affirmation 3: If $N$ is a riemannian manifold and $f$ is an isometric immersion, then $g$ is also an immersion. 
In deed, calculating $\mathrm{d} g(x, \xi)\left(v_{1}, v_{2}\right)$ we get

$$
\mathrm{d} g(x, \xi)\left(v_{1}, v_{2}\right)=\left\langle\mathrm{d} f(x) v_{1}, X_{2}\right\rangle \xi+\left\langle f(x), X_{2}\right\rangle v_{2}+\pi\left(\mathrm{d} f(x) v_{1}\right),
$$

where $X_{2} \in \mathbb{R}_{t-s}^{q+1}$ is a lightlike vector such that $\left\langle X_{1}, X_{2}\right\rangle=1$.

If $\mathrm{d} g(x)\left(v_{1}, v_{2}\right)=0$, then $\left\langle\mathrm{d} f(x) v_{1}, X_{2}\right\rangle \xi+\left\langle f(x), X_{2}\right\rangle v_{2}=0$ and $\pi\left(\mathrm{d} f(x) v_{1}\right)=0$, since $\xi, v_{2} \in$ $\mathbb{R}_{t-s}^{q+1} \perp \mathbb{R}_{s}^{n-q-1}$ and $\pi(f(x)) \in \mathbb{R}_{s}^{n-q-1}$.

Knowing that $N$ is riemannian and $f$ is an isometric immersion, we have that $\mathrm{d} f(x) v_{1}$ is null or it is a spacelike vector. But $\mathrm{d} f(x) v_{1}=\left\langle\mathrm{d} f(x) v_{1}, X_{2}\right\rangle X_{1}+\pi\left(\mathrm{d} f(x) v_{1}\right)=\left\langle\mathrm{d} f(x) v_{1}, X_{2}\right\rangle X_{1}$, ie., $\mathrm{d} f(x) v_{1}$ is not spacelike. Therefore $\mathrm{d} f(x) v_{1}=0$ and $v_{1}=0$.

Thus, $\mathrm{d} g(x, \xi)\left(v_{1}, v_{2}\right)=f_{1}(x) v_{2}=0$ and $g$ is an immersion, cause $f(N) \cap \mathbb{R}_{s}^{n-q-1}=\varnothing$ and $f_{1}(x) \neq 0 . \checkmark$

Remark 4. In case (I.3), through the calculations of the differential $\mathrm{d} g(x, \xi)$, it is easily proved that $g$ is an immersion if, and only if, $f_{*} T N \cap \operatorname{span}\left\{X_{1}\right\}=\{0\} \Leftrightarrow \mathbb{R}^{n-q} \cap\left(\mathbb{R}^{n-q}\right)^{\perp} \cap f_{*}(T N)=\{0\}$. Therefore, instead of supposing that $N$ is riemannian and $f$ is an isometric immersion, we could suppose that $f_{*} T N \cap \operatorname{span}\left\{X_{1}\right\}=\{0\}$, without changing the thesis.

We need more results in order to show case (II) of Proposition 2.

Let $X_{1}$ and $X_{2}$ be lightlike vectors of $\mathbb{R}_{t}^{n}$ such that $\left\langle X_{1}, X_{2}\right\rangle=1$ and lets suppose that

$$
\mathbb{R}_{t}^{n}=\operatorname{span}\left\{X_{1}, X_{2}\right\} \oplus U \oplus V,
$$

where $U$ and $V$ are nondegenerate vector subspaces. Lets consider the lightlike vector subspace $W:=\operatorname{span}\left\{X_{2}\right\} \oplus U \subset \mathbb{R}_{t}^{n}, \mathrm{O}(V)$ the group of linear isometries of $V$ and $\mathrm{O}(V) \ltimes V$ the group of isometries of $V$. We can define the applications $\mathscr{I}: V \rightarrow \operatorname{span}\left\{X_{1}, X_{2}\right\} \oplus V$ and $\Phi: \mathrm{O}(V) \ltimes V \rightarrow$ $\mathrm{O}_{t}(n)$ by

$$
\begin{aligned}
& \mathscr{I}(x):=X_{1}+x-\frac{\|x\|^{2}}{2} X_{2} \text { and } \\
& \Phi(B, x)\left(v+v^{\perp}\right):=v^{\perp}-\left(\langle B v, x\rangle+\frac{\left\langle X_{2}, v^{\perp}\right\rangle}{2}\|x\|^{2}\right) X_{2}+B v+\left\langle X_{2}, v^{\perp}\right\rangle x,
\end{aligned}
$$

for all $v+v^{\perp} \in V \oplus V^{\perp}=\mathbb{R}_{t}^{n}$.

In [8], it is proved the following lemma:

Lemma 5. $\quad 1 . \mathscr{I}: V \rightarrow \mathscr{I}(V)$ is an isometry.

2. $\Phi: \mathrm{O}(V) \ltimes V \rightarrow \mathscr{W}$ is a group isomorphism, where $\mathscr{W}$ is the subgroup of $\mathrm{O}_{t}(n)$ which fixes the points of $W$.

3. $\mathscr{W}$ is the isometries group of $\mathscr{I}(V)=\left\{X_{1}+x-\frac{\|x\|^{2}}{2} X_{2} \mid x \in V\right\}$.

Proof of the case (II) of Proposition 2.

Lets suppose that $\mathbb{R}^{n-q-1}$ is lightlike and that there exist a nondegenerate vector subspace $U \subset \mathbb{R}_{t}^{n}$ and a lightlike vector $X_{2} \in \mathbb{R}_{t}^{n}$ such that $\mathbb{R}^{n-q-1}=\operatorname{span}\left\{X_{2}\right\} \oplus U$. In this case, there exist a lightlike vector $X_{1} \in \mathbb{R}_{t}^{n}$ and a nondegenerate subspace $V \subset \mathbb{R}_{t}^{n}$ such that

$$
\mathbb{R}_{t}^{n}=\operatorname{span}\left\{X_{1}, X_{2}\right\} \oplus U \oplus V, \quad\left\langle X_{1}, X_{2}\right\rangle=1 \quad \text { and } \quad \mathbb{R}^{n-q}=\operatorname{span}\left\{w, X_{2}\right\} \oplus U,
$$


where $w \in V$, or $w=X_{1}$.

If $A \in \mathrm{O}(q+1)$ and $x \in N$, then

$$
A(f(x))=A\left(f_{1}(x) w+\pi(f(x))\right)=f_{1}(x) A(w)+\pi(f(x)) .
$$

By Lemma 5, there exist an isometry $B$ of $V$ and a vector $v \in V$ such that $A=\Phi(B, v)$.

(II.1): Lets suppose that $\mathbb{R}^{n-q}=\mathbb{R}_{s}^{n-q}=\operatorname{span}\left\{X_{1}, X_{2}\right\} \oplus U$. In this case, $f_{1}(x)=\left\langle f(x), X_{2}\right\rangle$ and we can write $f(x)=f_{1}(x) X_{1}+\pi(f(x))$. Thus,

$$
A(f(x))=f_{1}(x) A\left(X_{1}\right)+\pi(f(x)) .
$$

By the other side,

$$
A\left(X_{1}\right)=\Phi(B, v)\left(X_{1}\right) \stackrel{(2.2)}{=} X_{1}-\frac{\|v\|^{2}}{2} X_{2}+v \Rightarrow A(f(x))=f_{1}(x)\left(X_{1}-\frac{\|v\|^{2}}{2} X_{2}+v\right)+\pi(f(x)) .
$$

Thus, $M \subset \bar{M}$.

Let $f_{1}(x)\left(X_{1}-\frac{\|v\|^{2}}{2} X_{2}+v\right)+\pi(f(x)) \in \bar{M}$. Given $B \in \mathrm{O}(V), \Phi(B, v) \in \mathrm{O}(q+1)$, by Lemma 5. Furthermore, $\Phi(B, v)\left(X_{1}\right)=X_{1}-\frac{\|v\|^{2}}{2} X_{2}+v$, thus

$$
f_{1}(x)\left(X_{1}-\frac{\|v\|^{2}}{2} X_{2}+v\right)+\pi(f(x))=f_{1}(x) \Phi(B, v)\left(X_{1}\right)+\pi(f(x))=\Phi(B, v)(f(x)) \in M .
$$

Therefore, $M=\bar{M}$.

Calculating $\mathrm{d} g(x, v)$ we get

$$
\begin{array}{r}
\mathrm{d} g(x, v)\left(v_{1}, v_{2}\right)=\left\langle\mathrm{d} f(x) v_{1}, X_{2}\right\rangle X_{1}-\left(\left\langle\mathrm{d} f(x) v_{1}, X_{2}\right\rangle \frac{\|v\|^{2}}{2}+f_{1}(x)\left\langle v, v_{2}\right\rangle\right) X_{2}+\pi\left(\mathrm{d} f(x) v_{1}\right)+ \\
+\left\langle\mathrm{d} f(x) v_{1}, X_{2}\right\rangle v+f_{1}(x) v_{2}
\end{array}
$$

If $\mathrm{d} g(x, v)\left(v_{1}, v_{2}\right)=0$, then

$$
\left\{\begin{array}{l}
\left\langle\mathrm{d} f(x) v_{1}, X_{2}\right\rangle X_{1}=0 \Rightarrow\left\langle\mathrm{d} f(x) v_{1}, X_{2}\right\rangle=0 \\
\left\langle\mathrm{~d} f(x) v_{1}, X_{2}\right\rangle v+f_{1}(x) v_{2}=0 \Rightarrow f_{1}(x) v_{2}=0 \Rightarrow v_{2}=0 \\
-\left(f_{1}(x)\left\langle v, v_{2}\right\rangle+\left\langle\mathrm{d} f(x) v_{1}, X_{2}\right\rangle \frac{\|v\|^{2}}{2}\right) X_{2}+\pi\left(\mathrm{d} f(x) v_{1}\right)=0 \Rightarrow \pi\left(\mathrm{d} f(x) v_{1}\right)=0
\end{array}\right.
$$

since $v, v_{2} \in V \perp \mathbb{R}^{n-q}, \mathbb{R}^{n-q}=\operatorname{span}\left\{X_{1}, X_{2}\right\} \oplus U$ and $\pi(f(x)) \in \mathbb{R}^{n-q-1}=\operatorname{span}\left\{X_{2}\right\} \oplus U$. Therefore $f$ is an immersion. •

(II.2): Lets suppose that $\mathbb{R}^{n-q}=\operatorname{span}\{w\} \oplus \mathbb{R}^{n-q-1}=\operatorname{span}\left\{w, X_{2}\right\} \oplus U$, for some unit vector $w \in V$. In this case, $f_{1}=\varepsilon\langle f(x), w\rangle$, where $\varepsilon=\|w\|^{2}$. Thus,

$$
A(f(x))=f_{1}(x) \Phi(B, v)(w)+\pi(f(x)) \stackrel{(2.2)}{=} f_{1}(x)\left(-\langle B w, v\rangle X_{2}+B w\right)+\pi(f(x)) .
$$

Calling $\lambda:=-\langle B w, v\rangle$, we have that $M \subset \bar{M}$, since $\|B w\|^{2}=\|w\|^{2}$. Lets consider $f_{1}(x)\left(\lambda X_{2}+\right.$ $\xi)+\pi(f(x)) \in \bar{M}, B \in \mathrm{O}(V)$ and $v \in V$ such that $B w=\xi$ and $\langle\xi, v\rangle=-\lambda$, in this way

$$
\begin{aligned}
& f_{1}(x)\left(\lambda X_{2}+\xi\right)+\pi(f(x))=f_{1}(x)\left(-\langle B w, v\rangle X_{2}+B w\right)+\pi(f(x))= \\
& =f_{1}(x) \Phi(B, v)(w)+\pi(f(x))=\Phi(B, v)(f(x)) .
\end{aligned}
$$


Therefore $f_{1}(x)\left(\lambda X_{2}+\xi\right)+\pi(f(x)) \in M$.

Calculating $\mathrm{d} g(x, \xi, \lambda)$ we get

$$
\begin{aligned}
\mathrm{d} g(x, \xi, \lambda)\left(v_{1}, v_{2}, r\right)=\left[\varepsilon\left\langle\mathrm{d} f(x) v_{1}, w\right\rangle \xi+f_{1}(x) v_{2}\right]+\left[\varepsilon\left\langle\mathrm{d} f(x) v_{1}, w\right\rangle \lambda+f_{1}(x) r\right] & X_{2}+ \\
& +\pi\left(\mathrm{d} f(x) v_{1}\right) .
\end{aligned}
$$

If $\mathrm{d} g(x, \xi, \lambda)\left(v_{1}, v_{2}, r\right)=0$, then

$$
\left\{\begin{array}{l}
\varepsilon\left\langle\mathrm{d} f(x) v_{1}, w\right\rangle \xi+f_{1}(x) v_{2}=0 \\
{\left[\varepsilon\left\langle\mathrm{d} f(x) v_{1}, w\right\rangle \lambda+f_{1}(x) r\right] X_{2}+\pi\left(\mathrm{d} f(x) v_{1}\right)=0,}
\end{array}\right.
$$

since $\xi, v_{2} \in V$ and $X_{2}, \pi\left(\mathrm{d} f(x) v_{1}\right) \in V^{\perp}$.

In this way, $\left\langle\mathrm{d} f(x) v_{1}, w\right\rangle=0$ and $v_{2}=0$, since $\xi \in \mathbb{S}(0, \varepsilon), v_{2} \perp \mathbb{S}(0, \varepsilon)$ and $f(x) \notin \mathbb{R}^{n-q-1}$, thus $f_{1}(x) r X_{2}+\pi\left(\mathrm{d} f(x) v_{1}\right)=0$. If $f$ is an isometric immersion and $N$ is riemannian, then $g$ is an immersion. •

Remark 6. Using the calculations above for the case (II.2) of Proposition 2,

$$
\mathrm{d} g(x, \xi, \lambda)\left(v_{1}, v_{2}, r\right)=0 \Leftrightarrow\left\{\begin{array}{l}
\left\langle\mathrm{d} f(x) v_{1}, w\right\rangle=0, \\
v_{2}=0, \\
f_{1}(x) r X_{2}+\pi\left(\mathrm{d} f(x) v_{1}\right)=0
\end{array}\right.
$$

Therefore, $g$ is an immersion if, and only if, $f_{*}(T N) \cap \operatorname{span}\left\{X_{2}\right\}=\{0\} \Leftrightarrow \mathbb{R}^{n-q} \cap\left(\mathbb{R}^{n-q}\right)^{\perp} \cap$ $f_{*}(T N)=\{0\}$.

Definition 7. The immersion g given at Proposition 2 is called rotational immersion of the rotational submanifold $M$.

Proof of Corollary 3. Let $f: N^{m-q} \rightarrow \mathbb{R}^{n-q} \subset \mathbb{L}^{n}$ be an immersion and $M$ a rotational submanifold on $f$ with axis $\mathbb{R}^{n-q-1} \subset \mathbb{R}^{n-q}$. The only possibilities we have for $\mathbb{R}^{n-q-1}$ and $\mathbb{R}^{n-q}$ are:

1. $\mathbb{R}^{n-q-1}$ and $\mathbb{R}^{n-q}$ are both spacelike or both timelike, ie., both have the same index (equals to \pm 1 );

2. $\mathbb{R}^{n-q-1}$ is spacelike and $\mathbb{R}^{n-q}$ is timelike, ie., $\mathbb{R}^{n-q-1}$ has index 0 and $\mathbb{R}^{n-q}$ has index 1 ;

3. $\mathbb{R}^{n-q-1}$ is spacelike and $\mathbb{R}^{n-q}$ is lightlike;

4. $\mathbb{R}^{n-q-1}$ is lightlike and $\mathbb{R}^{n-q}$ is timelike;

5. $\mathbb{R}^{n-q-1}$ and $\mathbb{R}^{n-q}$ are both lightlike.

But all cases above were studied by Proposition 2.

Remarks 8. By observations 4 and 6 , if $M$ is a rotational submanifold in $\mathbb{L}^{n}$ on $f: N \rightarrow \mathbb{R}^{n-q}$ and $\mathbb{R}^{n-q}$ is lightlike, then $g$ is an immersion if, and only if, $\mathbb{R}^{n-q} \cap\left(\mathbb{R}^{n-q}\right)^{\perp} \cap \varphi_{*}(T N)=\{0\}$, ie., $g$ is an immersion if, and only if, $N$ is a riemannian manifold with the metric induced by $f$. 


\section{Proof of Theorem 1}

In order to prove Theorem 1, we need some additional results. The euclidean versions of these results can be found in [5].

Lemma 9. Let $f: M^{m} \rightarrow \mathbb{R}_{t}^{n}$ be an isometric immersion and $\eta$ a principal normal of $f$. Then, for all $X \in E_{\eta}(x)$ and all $\xi, \zeta \in T_{x}^{\perp} M$ such that $\xi \perp \eta$ and $\langle\zeta, \eta\rangle=1$, the following formulas are true:

$$
A_{\eta} X=\|\eta\|^{2} X, \quad A_{\xi} X=0 \quad \text { e } \quad A_{\zeta} X=X .
$$

Let $\mathscr{D}$ be a distribution in $M$ such that $\mathscr{D}(x) \subset E_{\eta}(x)$, for all $x \in M$.

1. If $\eta$ is parallel in the normal connexion of $f$ along $\mathscr{D}$, then $\nabla\|\eta\|^{2} \in \Gamma\left(\mathscr{D}^{\perp}\right)$, where $\nabla\|\eta\|^{2}$ is the gradient vector of $\|\eta\|^{2}$. Furthermore, the following formulas are true:

$$
\begin{aligned}
& \left(\|\eta\|^{2} \operatorname{Id}-A_{\eta}\right) \nabla_{X} Y=\frac{\langle X, Y\rangle}{2} \nabla\|\eta\|^{2}, \\
& \left\langle A_{\xi} \nabla_{X} Y, Z\right\rangle=\langle X, Y\rangle\left\langle\nabla_{Z}^{\perp} \xi, \eta\right\rangle, \\
& \left\langle\left(\operatorname{Id}-A_{\zeta}\right) \nabla_{X} Y, Z\right\rangle=-\langle X, Y\rangle\left\langle\nabla_{Z}^{\perp} \zeta, \eta\right\rangle,
\end{aligned}
$$

for all $X, Y \in \Gamma(\mathscr{D})$, all $Z \in \Gamma\left(\mathscr{D}^{\perp}\right)$ an all $\xi, \zeta \in \Gamma\left(T^{\perp} M\right)$ such that $\xi \perp \eta$ and $\langle\zeta, \eta\rangle=1$.

2. If $\mathscr{D}$ is an umbilical distribution and $\varphi$ is its mean curvature vector, then

$$
\tilde{\nabla}_{X} f_{*} Y=f_{*} \nabla_{X}^{v} Y+\langle X, Y\rangle \sigma, \forall X, Y \in \Gamma(\mathscr{D}),
$$

where $\sigma:=f_{*} \varphi+\eta$ and $\nabla_{X}^{v} Y$ is the orthogonal projection of $\nabla_{X} Y$ on $\mathscr{D}$.

3. With the same hypothesis of (I) and (II),

$$
\begin{aligned}
& \left(\|\eta\|^{2} \mathrm{Id}-A_{\eta}\right) \varphi=\frac{1}{2} \nabla\left(\|\eta\|^{2}\right), \\
& \left\langle A_{\xi} \varphi, Z\right\rangle=\left\langle\nabla_{Z}^{\perp} \xi, \eta\right\rangle, \\
& \left\langle\left(\mathrm{Id}-A_{\zeta}\right) \varphi, Z\right\rangle=-\left\langle\nabla_{Z}^{\perp} \zeta, \eta\right\rangle, \\
& \left\langle\nabla_{X} \varphi,\left(\|\eta\|^{2} \mathrm{Id}-A_{\eta}\right) Z\right\rangle=0, \\
& \left\langle\nabla_{X} \varphi, A_{\xi} Z\right\rangle=0, \\
& \left\langle\nabla_{X} \varphi,\left(\mathrm{Id}-A_{\zeta}\right) Z\right\rangle=0,
\end{aligned}
$$

for all $X \in \Gamma(\mathscr{D})$, all $Z \in \Gamma\left(\mathscr{U}^{\perp}\right)$ and all $\xi, \zeta \in \Gamma\left(T^{\perp} M\right)$ such that $\xi \perp \eta e\langle\zeta, \eta\rangle=1$.

Proof. Let $X \in E_{\eta}(x), Y \in T_{x} M$ and $\xi, \zeta \in T_{x}^{\perp} M$ such that $\xi \perp \eta$ and $\langle\zeta, \eta\rangle=1$. Then

$$
\begin{aligned}
& \left\langle A_{\eta} X, Y\right\rangle=\langle\alpha(X, Y), \eta\rangle=\langle\langle X, Y\rangle \eta, \eta\rangle=\|\eta\|^{2}\langle X, Y\rangle, \\
& \left\langle A_{\xi} X, Y\right\rangle=\langle\alpha(X, Y), \xi\rangle=\langle X, Y\rangle\langle\eta, \xi\rangle=0, \\
& \left\langle A_{\zeta} X, Y\right\rangle=\langle\alpha(X, Y), \zeta\rangle=\langle X, Y\rangle\langle\eta, \zeta\rangle=\langle X, Y\rangle .
\end{aligned}
$$

Therefore $A_{\eta} X=\|\eta\|^{2} X, A_{\xi} X=0$ e $A_{\zeta} X=X$. 
Let $X, Y \in \Gamma(\mathscr{D}), Z \in \Gamma\left(\mathscr{D}^{\perp}\right)$ and $\xi, \zeta \in \Gamma\left(T_{f}^{\perp} M\right)$ such that $\xi \perp \eta$ e $\langle\xi, \zeta\rangle=1$.

(I): Knowing that $\eta$ is parallel in the normal connection of $f$ along $\mathscr{D}$, then

$$
X\left(\|\eta\|^{2}\right)=0 \Rightarrow\left\langle X, \nabla\|\eta\|^{2}\right\rangle=0
$$

Therefore $\nabla\|\eta\|^{2} \in \Gamma\left(\mathscr{D}^{\perp}\right)$.

Using the Codazzi Equation and equation (3.1), and after some computations, we get that

$$
\nabla_{X} A_{\eta} Z-A_{\eta} \nabla_{X} Z=Z\left(\|\eta\|^{2}\right) X+\left(\|\eta\|^{2} \mathrm{Id}-A_{\eta}\right) \nabla_{Z} X-A_{\nabla_{\bar{Z}} \eta} X
$$

Taking the inner product of both sides of the above equality by $Y$, and after some computations, we obtain

$$
\left\langle Z,\left(\|\eta\|^{2} \mathrm{Id}-A_{\eta}\right) \nabla_{X} Y\right\rangle=\frac{\langle X, Y\rangle}{2}\left\langle\nabla\|\eta\|^{2}, Z\right\rangle .
$$

We know that, if $K \in \mathscr{D}$, then $\left\langle\left(\|\eta\|^{2} \mathrm{Id}-A_{\eta}\right) \nabla_{X} Y, K\right\rangle=\left\langle\nabla_{X} Y,\left(\|\eta\|^{2} \mathrm{Id}-A_{\eta}\right) K\right\rangle=0$, that is, the only component of $\left(\|\eta\|^{2} \mathrm{Id}-A_{\eta}\right) \nabla_{X} Y$ is in $\mathscr{D}^{\perp}$. Therefore, equation (3.2) follows from equation (3.12).

We can derive Equation (3.3) making similar computations from Codazzi Equation for $A_{\xi}, X$ and $Z$ and taking the inner product with $Y$. Equation (3.4) is similar, but we must use $X, A_{\zeta}$ and $Z$ at Codazzi Equation.

(II): If $\mathscr{D}$ is an umbilical distribution and $\varphi$ is its mean curvature vector, then

$$
\begin{aligned}
& \tilde{\nabla}_{X} f_{*} Y=f_{*} \nabla_{X} Y+\alpha(X, Y)=f_{*} \nabla_{X}^{v} Y+f_{*} \nabla_{X}^{h} Y+\langle X, Y\rangle \eta= \\
& =f_{*} \nabla_{X}^{v} Y+\langle X, Y\rangle f_{*} \varphi+\langle X, Y\rangle \eta=f_{*} \nabla_{X}^{v} Y+\langle X, Y\rangle \sigma . \bullet
\end{aligned}
$$

(III): If $\mathscr{D}$ is an umbilical distribution and $\varphi$ is its mean curvature vector, then $\nabla_{X} X=\nabla_{X}^{v} X+$ $\nabla_{X}^{h} X$ e $\nabla_{X}^{h} X=\varphi$, where $\nabla_{X}^{v} X$ and $\nabla_{X}^{h} X$ are the orthogonal projections of $\nabla_{X} X$ on $\mathscr{D}$ and on $\mathscr{D}^{\perp}$, respectively. Thus,

$$
\begin{aligned}
& \left(\|\eta\|^{2} \mathrm{Id}-A_{\eta}\right) \varphi=\left(\|\eta\|^{2} \mathrm{Id}-A_{\eta}\right) \nabla_{X}^{h} X \stackrel{(3.1)}{=}\left(\|\eta\|^{2} \mathrm{Id}-A_{\eta}\right)\left(\nabla_{X}^{v} X+\nabla_{X}^{h} X\right)= \\
& =\left(\|\eta\|^{2} \mathrm{Id}-A_{\eta}\right) \nabla_{X} X \stackrel{(3.2)}{=} \frac{1}{2} \nabla\|\eta\|^{2}
\end{aligned}
$$

Therefore equation (3.6) is true.

The equations (3.7) and (3.8) follow, respectively, from equations (3.3) and (3.4), using equation (3.1).

Using (3.6), we can compute that

$$
\left\langle\nabla_{X} \varphi,\left(\|\eta\|^{2} \mathrm{Id}-A_{\eta}\right) Z\right\rangle=\frac{1}{2} X\left\langle\nabla\|\eta\|^{2}, Z\right\rangle-\left\langle\varphi, \nabla_{X}\left(\|\eta\|^{2} \mathrm{Id}-A_{\eta}\right) Z\right\rangle .
$$

Using Codazzi Equation for $A_{\eta}, X$ and $Z$, using equation (3.6), and after some computations, we obtain

$$
\left\langle\nabla_{X}\left(\|\eta\|^{2} \mathrm{Id}-A_{\eta}\right) Z, \varphi\right\rangle=\frac{1}{2} X Z\left(\|\eta\|^{2}\right)=\frac{1}{2} X\left\langle Z, \nabla\|\eta\|^{2}\right\rangle .
$$

Thus we get the equation (3.9) replacing the last equation in (3.13). 
We know that $\eta$ is parallel in the normal connection of $f$ along $\mathscr{D}$ and $\xi \perp \eta$, thus $\left\langle\nabla_{X}^{\perp} \xi, \eta\right\rangle=$ $-\left\langle\xi, \nabla_{X}^{\perp} \eta\right\rangle=0$, that is, $\nabla_{X}^{\perp} \xi \perp \eta$. In this way, using the Codazzi Equation for $A_{\xi}, X$ and $Z$, using equation (3.7), and after some computations, we obtain

$$
\left\langle A_{\xi} Z, \nabla_{X} \varphi\right\rangle=\left\langle\mathscr{R}^{\perp}(X, Z) \xi, \eta\right\rangle .
$$

By the other side, by Ricci Equation,

$$
\left\langle\mathscr{R}^{\perp}(X, Z) \xi, \eta\right\rangle=\langle\tilde{\mathscr{R}}(X, Z) \xi, \eta\rangle-\left\langle\left[A_{\xi}, A_{\eta}\right] X, Z\right\rangle=0 .
$$

Therefore, the equation (3.10) is true.

Similarly, equation (3.11) is obtained using the Codazzi equation for $A_{\zeta}, X$ and $Z$, equations (3.7) and (3.8) and the Ricci Equation for $X, Z, \zeta$ and $\eta$ •

Corollary 10. Let $f: M^{m} \rightarrow \mathbb{R}_{t}^{n}$ be an isometric immersion. If $\eta$ is a non null Dupin normal of $f$, $E_{\eta}$ is an umbilical distribution and $\varphi$ is the mean curvature vector of $E_{\eta}$, then $E_{\eta}$ is a spherical distribution and the equations of Lemma 9 are true.

Proof. Taking $\mathscr{D}:=E_{\eta}$, the formulas of Lemma 9 are true. To show that $E_{\eta}$ is spherical, we will show that $\nabla_{X} \varphi(x) \in E_{\eta}(x)$, for all $x \in M$ and all $X \in E_{\eta}(x)$. But this is equivalent to show that

$$
\left(A_{\psi}-\langle\psi, \eta\rangle \mathrm{Id}\right) \nabla_{X} \varphi(x)=0
$$

for all $x \in M$ and all $\psi \in T_{x}^{\perp} M$.

Let $x \in M$ and $\psi \in T_{x}^{\perp} M$.

If $\eta(x)$ is timelike or spacelike.

In this case, $\|\eta(x)\|^{2} \neq 0$ and

$$
\begin{aligned}
& A_{\psi}-\langle\psi, \eta\rangle \mathrm{Id}=A_{\psi-\langle\psi, \eta\rangle \frac{\eta}{\|\eta\|^{2}}}+\langle\psi, \eta\rangle A_{\frac{\eta}{\|\eta\|^{2}}}-\langle\psi, \eta\rangle \mathrm{Id}= \\
& =A_{\psi-\langle\psi, \eta\rangle \frac{\eta}{\|\eta\|^{2}}}+\langle\psi, \eta\rangle\left(A_{\frac{\eta}{\|\eta\|^{2}}}-\mathrm{Id}\right)=A_{\xi}+\langle\psi, \eta\rangle\left(A_{\frac{\eta}{\|\eta\|^{2}}}-\mathrm{Id}\right)
\end{aligned}
$$

where $\xi:=\psi-\langle\psi, \eta\rangle \frac{\eta}{\|\eta\|^{2}} \perp \eta$. If $Z \in E_{\eta}^{\perp}(x)$, then

$$
\left\langle\left(A_{\psi}-\langle\psi, \eta\rangle \mathrm{Id}\right) \nabla_{X} \varphi, Z\right\rangle=\left\langle A_{\xi} \nabla_{X} \varphi, Z\right\rangle+\langle\psi, \eta\rangle\left\langle\left(A_{\frac{\eta}{\|\eta\|^{2}}}-\mathrm{Id}\right) \nabla_{X} \varphi, Z\right\rangle .
$$

By equations (3.9) e (3.10),

$$
\left\langle\nabla_{X} \varphi, A_{\xi} Z\right\rangle=0=\left\langle\nabla_{X} \varphi,\left(\|\eta\|^{2} \mathrm{Id}-A_{\eta}\right) Z\right\rangle .
$$

Therefore $\left\langle\left(A_{\psi}-\langle\psi, \eta\rangle\right.\right.$ Id $\left.) \nabla_{X} \varphi, Z\right\rangle=0$. It remains to prove that $\left\langle\left(A_{\psi}-\langle\psi, \eta\rangle\right.\right.$ Id $\left.) \nabla_{X} \varphi(x), Y\right\rangle=$ 0 , for all $Y \in E_{\eta}(x)$. But

$$
\left\langle\left(A_{\psi}-\langle\psi, \eta\rangle \mathrm{Id}\right) \nabla_{X} \varphi(x), Y\right\rangle=\left\langle\nabla_{X} \varphi, A_{\xi} Y\right\rangle+\langle\psi, \eta\rangle\left\langle\nabla_{X} \varphi,\left(A_{\frac{\eta}{\|\eta\|^{2}}}-\mathrm{Id}\right) Y\right\rangle \stackrel{(3.1)}{=} 0 .
$$


If $\eta(x)$ is non null and lightlike.

In this case, there exists a lightlike vector $\zeta \in T_{x}^{\perp} M$ such that $\langle\eta, \zeta\rangle=1$. Thus,

$$
A_{\psi}-\langle\psi, \eta\rangle \mathrm{Id}=A_{\psi-\langle\psi, \eta\rangle \zeta}+\langle\psi, \eta\rangle A_{\zeta}-\langle\psi, \eta\rangle \mathrm{Id}=A_{\xi}+\langle\psi, \eta\rangle\left(A_{\zeta}-\mathrm{Id}\right),
$$

where $\xi:=\psi-\langle\psi, \eta\rangle \zeta \perp \eta$.

If $Z \in E_{\eta}^{\perp}(x)$,

$$
\left\langle\left(A_{\psi}-\langle\psi, \eta\rangle \mathrm{Id}\right) \nabla_{X} \varphi, Z\right\rangle=\left\langle A_{\xi} \nabla_{X} \varphi, Z\right\rangle+\langle\psi, \eta\rangle\left\langle\left(A_{\zeta}-\mathrm{Id}\right) \nabla_{X} \varphi(x), Z\right\rangle .
$$

By the equalities (3.10) e (3.11),

$$
\left\langle\nabla_{X} \varphi, A_{\xi} Z\right\rangle=0=\left\langle\nabla_{X} \varphi,\left(\mathrm{Id}-A_{\zeta}\right) Z\right\rangle
$$

Therefore $\left\langle\left(A_{\psi}-\langle\psi, \eta\rangle\right.\right.$ Id $\left.) \nabla_{X} \varphi, Z\right\rangle=0$.

By the other side, if $Y \in E_{\eta}(x)$,

$$
\left\langle\left(A_{\psi}-\langle\psi, \eta\rangle \mathrm{Id}\right) \nabla_{X} \varphi, Y\right\rangle=\left\langle\nabla_{X} \varphi, A_{\xi} Y\right\rangle+\langle\psi, \eta\rangle\left\langle\nabla_{X} \varphi,\left(A_{\zeta}-\mathrm{Id}\right) Y\right\rangle \stackrel{(3.1)}{=} 0 .
$$

Proposition 11. Let $M^{m}$ be a riemannian manifold, $f: M^{m} \rightarrow \mathbb{R}_{t}^{n}$ an isometric immersion and $\eta$ its non null principal normal.

1. If $\operatorname{dim} E_{\eta}$ is constant and $\operatorname{dim} E_{\eta} \geq 2$, then $\eta$ is parallel in the normal connexion of $f$ along $E_{\eta}$, ie., $\eta$ is a Dupin normal.

2. If $\mathscr{D} \subset E_{\eta}$ is a spherical distribution in $M$ whose leafs are open subsets of

(a) q-dimensional ellipsoids given by the intersection $\mathbb{S}(c, r) \cap(c+L) \subset \mathbb{R}_{t}^{n}$, where L is a spacelike $(q+1)$-dimensional vector of $\mathbb{R}_{t}^{n}$;

(b) or q-dimensional hyperboloids given by the intersection $\mathbb{S}(c,-r) \cap(c+L) \subset \mathbb{R}_{t}^{n}$, where $L$ is a timelike $(q+1)$-dimensional vector of $\mathbb{R}_{t}^{n}$;

(c) or q-dimensional paraboloids given by $\left[\mathscr{L}_{*} \cap(c+L)\right]+d \subset \mathbb{R}_{t}^{n}$, where $L=\operatorname{span}\{w\} \oplus$ $V$ is a lightlike $(q+1)$-dimensional vector of $\mathbb{R}_{t}^{n}$ (with $V$ spacelike and $w$ lightlike), $c \perp V$ is lightlike and $\langle c, w\rangle \neq 0$;

then $\eta$ is parallel in the normal connexion of $f$ along $\mathscr{D}$.

3. If $\eta$ Dupin normal with multiplicity $q$, then $E_{\eta}$ is an spherical distribution in $M^{m}$.

In this case, let $x \in M, N$ be a leaf of $E_{\eta}$ with $x \in N$ and $\sigma:=f_{*} \varphi+\eta$, where $\varphi$ is the mean curvature vector of $E_{\eta}$.

(a) If $\sigma(x)$ is spacelike, then $f(N)$ is an open subset of a q-dimensional ellipsoid in $\mathbb{R}_{t}^{n}$ given by the intersection $\mathbb{S}(c, r) \cap(c+L)$, where $L$ is a spacelike $(q+1)$-dimensional subspace of $\mathbb{R}_{t}^{n}$.

(b) If $\sigma(x)$ is timelike, then $f(N)$ is an open subset of a q-dimensional hyperboloid in $\mathbb{R}_{t}^{n}$ given by the intersection $\mathbb{S}(c,-r) \cap(c+L)$, where $L$ is a timelike $(q+1)$-dimensional subspace of $\mathbb{R}_{t}^{n}$. 
(c) If $\sigma(x)$ is lightlike and non null, then $f(N)$ is an open subset of a q-dimensional paraboloid in $\mathbb{R}_{t}^{n}$ given by $c+\left\{v+\frac{\|v\|^{2}}{2} w \mid v \in V(x)\right\}$, where $V \subset \mathbb{R}_{t}^{n}$ is a spacelike $q$-dimensional vector subspace and $w \perp V$ is lightlike.

Remarks 12. Through the proof made ahead, at the items (III.1) and (III.2) of Proposition 11,

$$
c=f(x)+\frac{\sigma(x)}{\|\sigma(x)\|^{2}}, \quad r=\frac{1}{\sqrt{\left|\|\sigma(x)\|^{2}\right|}} \quad \text { and } \quad L(x)=f_{*} E_{\eta}(x) \oplus \operatorname{span}\{\sigma(x)\}
$$

are constant in each leaf of $E_{\eta}$.

At the item (III.3), the paraboloids containing the leafs of $E_{\eta}$ are given by

$$
\begin{gathered}
p(x)+(-\tilde{\sigma}(x)+L) \cap \mathscr{L}=p(x)-\tilde{\sigma}(x)+\left\{v+\frac{\|v\|^{2}}{2} \sigma(x) \mid v \in V(x)\right\}, \\
\xi(x):=-\sum_{i=1}^{q}\left\langle\mathrm{~d} f(x) e_{i}, \tilde{\sigma}(x)\right\rangle \mathrm{d} f(x) e_{i}+\frac{1}{2} \sum_{i=1}^{q}\left\langle\mathrm{~d} f(x) e_{i}, \tilde{\sigma}(x)\right\rangle^{2} \sigma(x)+\tilde{\sigma}(x) .
\end{gathered}
$$

Proof of Proposition 11.

Let $X^{v}$ and $X^{h}$ be the orthogonal projections of $X \in \Gamma(T M)$ on $\mathscr{D}$ and $\mathscr{D}^{\perp}$, respectively. Likewise, let $\nabla_{X}^{v} Y$ and $\nabla_{X}^{h} Y$ be the orthogonal projections of $\nabla_{X} Y$ on $\mathscr{D}$ and $\mathscr{D}^{\perp}$, respectively.

(I): Let $\mathscr{D}:=E_{\eta}, X, Y \in \Gamma\left(E_{\eta}\right)$ and $\xi, \zeta \in \Gamma\left(T^{\perp} M\right)$ such that $\xi \perp \eta$ e $\langle\zeta, \eta\rangle=1$. By Codazzi Equation for $A_{\xi}, X$ and $Y$ and using (3.1), we get

$$
A_{\xi} \nabla_{X} Y+A_{\nabla_{X} \xi} Y=A_{\xi} \nabla_{Y} X+A_{\nabla_{Y}} \xi X .
$$

We suppose that $X \perp Y$ and that $\|Y\|^{2}=1$, since $\operatorname{dim} E_{\eta} \geq 2$. Thus, taking the inner product with $Y$ of both sides of the above equation, using (3.1) and after some calculations, we can get that $\left\langle\nabla_{X}^{\perp} \eta, \xi\right\rangle=0$.

Similarly, by Codazzi Equation for $A_{\zeta}, X$ and $Y$, and taking the inner product with $Y$, we can compute that $\left\langle\nabla_{X}^{\perp} \eta, \zeta\right\rangle=0$.

We conclude that $\nabla_{X}^{\perp} \eta=0$, cause $\left\langle\nabla_{X}^{\perp} \eta, \xi\right\rangle=0$ and $\left\langle\nabla_{X}^{\perp} \eta, \zeta\right\rangle=0$, for all $\xi, \zeta \in \Gamma\left(T^{\perp} M\right)$ such that $\xi \perp \eta$ and $\langle\zeta, \eta\rangle=1$. •

(II.1) and (II.2): Lets suppose that the leafs of $\mathscr{D}$ are open subsets of $q$-dimensional ellipsoids or hyperboloids given by $\mathbb{S}(c, \varepsilon r) \cap(c+L) \subset \mathbb{R}_{t}^{n}$, where

a) or $\varepsilon=1$ and $L$ is an $(q+1)$-dimensional spacelike subspace of $\mathbb{R}_{t}^{n}$, if $L$ is spacelike;

b) or $\varepsilon=-1$ and $L$ is and $(q+1)$-dimensional timelike subspace of $\mathbb{R}_{t}^{n}$, if $L$ is timelike.

Let $N \subset M$ be a leaf (integral submanifold) of $\mathscr{D}$. Thus, $f(N) \subset \mathbb{S}(c, \varepsilon r) \cap(c+L) \subset \mathbb{R}_{t}^{n}$, for some $c \in \mathbb{R}_{t}^{n}, r>0$ and some $(q+1)$-dimensional spacelike or timelike vector subspace $L^{q+1} \subset \mathbb{R}_{t}^{n}$. Lets define the field $\sigma: N \rightarrow \mathbb{R}_{t}^{n}$ by $\sigma(x):=-\varepsilon \frac{f(x)-c}{r^{2}}$ and let $X \in \Gamma(\mathscr{D})$, in this way

$$
\begin{aligned}
& \|\sigma\|^{2}=\frac{\varepsilon^{2}}{r^{4}}\|f(x)-c\|^{2}=\frac{\varepsilon^{3} r^{2}}{r^{4}}=\frac{\varepsilon}{r^{2}} \text { and } \\
& \left\langle\sigma, f_{*} X\right\rangle=-\varepsilon\left\langle\frac{f(x)-c}{r^{2}}, f_{*} X\right\rangle=-\varepsilon r^{2}\left\langle-\varepsilon \frac{f(x)-c}{r^{2}},-\varepsilon \frac{f_{*} X}{r^{2}}\right\rangle=-r^{2} \varepsilon\left\langle\sigma, \sigma_{*} X\right\rangle=0,
\end{aligned}
$$


that is, $\sigma$ is normal to $N$ and $\|\sigma\|^{2}=\frac{\varepsilon}{r^{2}}$ is constant in $N$.

Knowing that $\mathscr{D} \subset E_{\eta}$ and that $\mathscr{D}$ is a spherical distribution, we can get that

$$
\tilde{\nabla}_{X} f_{*} Y=f_{*} \nabla_{X}^{v} Y+\langle X, Y\rangle\left(f_{*} \varphi+\eta\right) .
$$

By the other side, $c+L$ is totally geodesic if $\mathbb{R}_{t}^{n}, f(N) \subset \mathbb{S}(c, \varepsilon r) \cap(c+L) \subset \mathbb{R}_{t}^{n}$ and $\nabla^{v}$ is the Levi-Civita connection of $N$, then

$$
\tilde{\nabla}_{X} f_{*} Y=f_{*} \nabla_{X}^{v} Y-\langle X, Y\rangle \varepsilon \frac{f-c}{r^{2}}=f_{*} \nabla_{X}^{v} Y+\langle X, Y\rangle \sigma
$$

Comparing the last two equations, we get that $\sigma=f_{*} \varphi+\eta$ and $\eta=\sigma-f_{*} \varphi$. Thus,

$$
\begin{aligned}
& \tilde{\nabla}_{X} \eta=\tilde{\nabla}_{X} \sigma-\tilde{\nabla}_{X} f_{*} \varphi=-\tilde{\nabla}_{X} \varepsilon \frac{f-c}{r^{2}}-f_{*} \nabla_{X} \varphi-\underline{\alpha}(X, \varphi)= \\
& =-\frac{\varepsilon}{r^{2}} f_{*} X-f_{*} \nabla_{X} \varphi, \text { cause } X \in \mathscr{D} \subset E_{\eta} .
\end{aligned}
$$

Therefore $\nabla \frac{1}{X} \eta=0$. •

(II.3): Lets suppose that the leafs of $\mathscr{D}$ are open subsets of $q$-dimensional paraboloids given by $[\mathscr{L} \cap(L+c)]+d \subset \mathbb{R}_{t}^{n}$, where $L=\operatorname{span}\{w\} \oplus V$ is a $(q+1)$-dimensional lightlike vector subspace of $\mathbb{R}_{t}^{n}$ (with $V$ spacelike and $w$ lightlike), $c \perp V$ is lightlike and $\langle c, w\rangle \neq 0$.

Let $N$ be a leaf of $\mathscr{D}$. But $[\mathscr{L} \cap(L+c)]+d \subset \operatorname{span}\{c, w\} \oplus V+d \subset \mathbb{R}_{t}^{n}$ and $\operatorname{span}\{c, w\} \oplus V+d$ is totally geodesic in $\mathbb{R}_{t}^{n}$, thus we can consider $\left.f\right|_{N}: N \rightarrow \operatorname{span}\{c, w\} \oplus V+d$.

But $f-d \in \mathscr{L}$, thus $f-d$ is field normal to $N$. Let $\left\{w, X_{1}, \cdots, X_{q}\right\}$ be a basis of $L$ such that $\left\{X_{1}, \cdots, X_{q}\right\}$ is a orthonormal basis of $V$. In this way, $\operatorname{span}\{c, w\} \oplus V=L+\operatorname{span}\{c\}=$ $\operatorname{span}\left\{w, \tilde{w}, X_{1}, \cdots, X_{q}\right\}$, where $\{w, \tilde{w}\}$ is a pseudo-orthonormal basis of $\operatorname{span}\{w, c\}$. We can suppose that $c=b \tilde{w}$.

We will show that $\left\langle f-d, \frac{w}{b}\right\rangle=1$. Indeed, $f(x)-d \in L+c$, thus

$$
f(x)-d=a(x) w+b \tilde{w}+\sum_{i=1}^{q} x_{i}(x) X_{i} \Rightarrow\left\langle f-d, \frac{w}{b}\right\rangle=1,
$$

and thus $w \perp N$.

But $f-d$ and $\frac{w}{b}$ are orthogonal to $N$ and $f(N) \subset \operatorname{span}\{c, w\} \oplus V+d$, then

$$
\begin{aligned}
& \alpha_{\left.f\right|_{N}}(X, Y)=\left\langle\alpha_{\left.f\right|_{N}}(X, Y), f-d\right\rangle \frac{w}{b}+\left\langle\alpha_{\left.f\right|_{N}}(X, Y), \frac{w}{b}\right\rangle(f-d)= \\
& =\left\langle A_{f-d} X, Y\right\rangle \frac{w}{b}+\left\langle A_{\frac{w}{b}} X, Y\right\rangle(f-d) .
\end{aligned}
$$

By the other side, $\tilde{\nabla}_{X} \frac{w}{b}=0$ and $\tilde{\nabla}_{X}(f-d)=f_{*} X$. Therefore $\alpha_{\left.f\right|_{N}}(X, Y)=-\langle X, Y\rangle \frac{w}{b}$.

By the same calculations made at the cases (II.1) and (II.2), we get that $\tilde{\nabla}_{X} f_{*} Y=f_{*} \nabla_{X}^{v} Y+$ $\langle X, Y\rangle\left(f_{*} \varphi+\eta\right)$. Thus

$$
\begin{aligned}
& -\frac{w}{b}=f_{*} \varphi+\eta \Rightarrow \eta=-\frac{w}{b}-f_{*} \varphi \Rightarrow \\
& \Rightarrow \tilde{\nabla}_{X} \eta=-\tilde{\nabla}_{X}\left(f_{*} \varphi\right)=-f_{*} \nabla_{X} \varphi-\left\langle X, \varphi \nmid \eta=-f_{*} \nabla_{X} \varphi .\right.
\end{aligned}
$$

Therefore $\nabla_{X}^{\perp} \eta=0$, for all $X \in \mathscr{D}$. • 
(III): If $\mathscr{D}:=E_{\eta}$, then, by Lemma 9, the equations (3.1) to (3.4) hold.

Affirmation 1: If $X, Y \in \Gamma\left(E_{\eta}\right)$ and $X \perp Y$, then $\nabla_{X} Y \in \Gamma\left(E_{\eta}\right)$.

If $Z \in \Gamma\left(E_{\eta}^{\perp}\right), \xi, \zeta \in \Gamma\left(T^{\perp} M\right), \xi \perp \eta$ and $\langle\zeta, \eta\rangle=1$, then

$$
\begin{aligned}
& \left(\|\eta\|^{2} \mathrm{Id}-A_{\eta}\right) \nabla_{X} Y \stackrel{(3.2)}{=} \frac{\langle X, Y\rangle}{2} \nabla\|\eta\|^{2}=0 \Rightarrow\|\eta\|^{2} \nabla_{X} Y=A_{\eta} \nabla_{X} Y ; \\
& \left\langle A_{\xi} \nabla_{X} Y, Z\right\rangle \stackrel{(3.3)}{=}\langle X, Y\rangle\left\langle\nabla_{Z} \xi, \eta\right\rangle=0 \Rightarrow A_{\xi} \nabla_{X} Y \in \Gamma\left(E_{\eta}\right) ; \\
& \left\langle\left(\operatorname{Id}-A_{\zeta}\right) \nabla_{X} Y, Z\right\rangle \stackrel{(3.4)}{=}-\langle X, Y\rangle\left\langle\nabla_{Z}^{\perp} \zeta, \eta\right\rangle=0 \Rightarrow\left(\operatorname{Id}-A_{\zeta}\right) \nabla_{X} Y \in \Gamma\left(E_{\eta}\right) .
\end{aligned}
$$

By the other side, if $W \in E_{\eta}$, then

$$
\left\{\begin{array}{l}
\left\langle A_{\xi} \nabla_{X} Y, W\right\rangle=\left\langle\nabla_{X} Y, A_{\xi} W\right\rangle \stackrel{(3.1)}{=} 0 ; \\
\left\langle\left(\operatorname{Id}-A_{\zeta}\right) \nabla_{X} Y, W\right\rangle=\left\langle\nabla_{X} Y,\left(\operatorname{Id}-A_{\zeta}\right) W\right\rangle \stackrel{(3.1)}{=} 0 .
\end{array}\right.
$$

Therefore

$$
A_{\xi} \nabla_{X} Y=0 \quad \text { e }\left(\mathrm{Id}-A_{\zeta}\right) \nabla_{X} Y=0
$$

for all $\xi, \zeta \in \Gamma\left(T^{\perp} M\right)$ such that $\xi \perp \eta$ and $\langle\zeta, \eta\rangle=1$.

Let $x \in M$ be a point and $\psi \in T_{x}^{\perp} M$ be a normal vector. If $\eta(x)$ is timelike or spacelike, then $\|\eta(x)\|^{2} \neq 0$, thus

$$
\left(A_{\psi}-\langle\psi, \eta\rangle \mathrm{Id}\right) \nabla_{X} Y=A_{\psi-\langle\psi, \eta\rangle \frac{\eta}{\|\eta\|^{2}}} \nabla_{X} Y-\langle\psi, \eta\rangle\left(\mathrm{Id}-A_{\frac{\eta}{\|\eta\|^{2}}}\right) \nabla_{X} Y \stackrel{(3.14),(3.15)}{=} 0 .
$$

If $\eta(x)$ is lightlike, then there exists a lightlike vector $\zeta \in T_{x}^{\perp} M$ such that $\langle\eta(x), \zeta\rangle=1$. In this case,

$$
\left(A_{\psi}-\langle\psi, \eta\rangle \mathrm{Id}\right) \nabla_{X} Y=A_{\psi-\langle\psi, \eta\rangle} \nabla_{X} Y-\langle\psi, \eta\rangle\left(\mathrm{Id}-A_{\zeta}\right) \nabla_{X} Y \stackrel{(3.14),(3.15)}{=} 0 .
$$

But $\left(A_{\psi}-\langle\psi, \eta\rangle \mathrm{Id}\right) \nabla_{X} Y=0$, for all $\psi$, is equivalent to $\nabla_{X} Y \in E_{\eta} \cdot \checkmark$

Affirmation 2: $E_{\eta}$ is umbilical.

We have to show that there exists $\varphi \in \Gamma\left(E_{\eta}^{\perp}\right)$ such that $\nabla_{X}^{h} Y=\langle X, Y\rangle \varphi$, for any pair of vector fields $X, Y \in \gamma\left(E_{\eta}\right)$. But the application $(X, Y) \mapsto \nabla_{X}^{h} Y$ is bilinear in $E_{\eta}$ because, for any $Z \in \Gamma\left(E_{\eta}^{\perp}\right),\left\langle\nabla_{X}^{h} Y, Z\right\rangle=-\left\langle Y, \nabla_{X} Z\right\rangle$. Besides that, Affirmation 1 stands that $X \perp Y \Rightarrow \nabla_{X}^{h} Y=0$. Then, a known Lemma stands that there exists $\varphi$ such that $\nabla_{X}^{h} Y=\langle X, Y\rangle \varphi$ (see, for example, Lemma A.9 in [9]).

If we take a unit differentiable vector field $X \in E_{\eta}$, then $\varphi=\nabla_{X}^{h} X$. Therefore $\varphi$ is differentiable. $\checkmark$

\section{Affirmation 3: $E_{\eta}$ is spherical and the equations from Lemma 9 hold.}

Just see Corollary 10. $\checkmark$

Let $N \subset M$ be a leaf of $E_{\eta}$ passing through $x$. Equation (3.5) stands that $\left.f\right|_{N}: N \rightarrow \mathbb{R}_{t}^{n}$ is an umbilical isometric immersion and that $\sigma$ is its mean curvature vector. Therefore, knowing the classifications of umbilical immersions in $\mathbb{R}_{t}^{n}$, we have that Remarks 12 hold and that

- or $f(N) \subset \mathbb{S}\left(c(x) ; \frac{1}{\|\sigma(x)\|}\right) \cap(c(x)+L(x))$, if $\sigma(x)$ is spacelike; 
- or $f(N) \subset \mathbb{S}\left(c(x) ;-\frac{1}{\|\sigma(x)\|}\right) \cap(c(x)+L(x))$, if $\sigma(x)$ is timelike;

- or $f(N) \subset p(x)+(-\tilde{\sigma}(x)+L(x)) \cap \mathscr{L}=p(x)-\tilde{\sigma}(x)+\left\{v+\frac{\|v\|^{2}}{2} \sigma(x): v \in V(x)\right\}$, if $\sigma(x)$ is lightlike.

For more details about umbilical immersions of a riemannian manifold in $\mathbb{R}_{t}^{n}$, see Chapter 1 of [9].

The following definition was given at [5]

Definition 13. Let $\mathscr{D}$ be an umbilical distribution in an riemannian manifold $M$. The splitting tensor $C$ of $\mathscr{D}$ is given by $C_{X} Z:=-\nabla_{Z}^{h} X$, for all $X \in \Gamma(\mathscr{D})$ and all $Z \in \Gamma\left(\mathscr{D}^{\perp}\right)$.

Remarks 14. Given an orthonormal frame $\left\{w_{1}, \cdots, w_{k}\right\}$ of $\mathscr{D}^{\perp}$, it follows that

$$
C_{X} Z=-\nabla_{Z}^{h} X=-\sum_{i=1}^{k}\left\langle\nabla_{Z}^{h} X, w_{i}\right\rangle w_{i}=\sum_{i=1}^{k}\left\langle X, \nabla_{Z} w_{i}\right\rangle w_{i} .
$$

Therefore $C_{f \cdot X} g \cdot Z=f \cdot g \cdot C_{X} Z$, for any pair of differentiable applications $f, g: M \rightarrow \mathbb{R}$, every $X \in \Gamma(\mathscr{D})$ and every $Z \in \Gamma\left(\mathscr{D}^{\perp}\right)$. Therefore $C$ is a tensor.

Lemma 15. Let $\mathscr{D}$ be an umbilical distribution in $M$ and $\varphi$ its mean curvature vector. If $X, Y \in \mathscr{D}$ and $W, Z \in \mathscr{D}^{\perp}$, then:

$$
\begin{aligned}
& \left(\nabla_{X}^{h} C_{Y}\right) W=C_{Y} C_{X} W+C_{\nabla_{X}^{v} Y} W-\mathscr{R}^{h}(X, W) Y+\langle X, Y\rangle\left(\langle W, \varphi\rangle-\nabla_{W}^{h} \varphi\right), \\
& \left(\nabla_{W}^{h} C_{X}\right) Z-\left(\nabla_{Z}^{h} C_{X}\right) W=C_{\nabla_{W}^{v} X} Z-C_{\nabla_{Z}^{v} X} W-\mathscr{R}^{h}(W, Z) X-\langle[W, Z], X\rangle \varphi,
\end{aligned}
$$

where $\mathscr{R}^{h}(X, W) Y$ is the orthogonal projection of $\mathscr{R}(X, W) Y$ on $\mathscr{D}^{\perp}$.

Se $\mathscr{D} \subset E_{\eta}$, then

$$
\begin{aligned}
& \left(\nabla_{X}^{h} C_{Y}\right) W=C_{Y} C_{X} W+C_{\nabla_{X}^{v} Y} W+\langle X, Y\rangle\left(A_{\eta} W+\langle W, \varphi\rangle \varphi-\nabla_{W}^{h} \varphi\right), \\
& \left(\nabla_{W}^{h} C_{X}\right) Z-\left(\nabla_{Z}^{h} C_{X}\right) W=C_{\nabla_{W}^{v} X} Z-C_{\nabla_{Z}^{v} X} W-\langle[W, Z], X\rangle \varphi .
\end{aligned}
$$

If $\eta$ is a principal normal of $f: M \rightarrow \mathbb{R} N, \mathscr{D} \subset E_{\eta}$ and $\mathscr{D}^{\perp}$ is a totally geodesic distribution, then

$$
\nabla_{W}^{h} \varphi=A_{\eta} W+\langle W, \varphi\rangle \varphi
$$

Proof. See Lemma 9 of [5], where it was first proved, or Lemma 2.15 of [9].

Now we can prove Theorem 1.

\section{Poof of Theorem 1.}

Taking $\mathscr{D}(x)=E_{\eta}(x)$, the items (I) of Lemma 9 and (III) of Proposition 11 stands that $\nabla\left(\|\eta\|^{2}\right) \in E_{\eta}^{\perp}$ and that $E_{\eta}$ is an spherical distribution. Let $\varphi$ be the mean curvature vector of $E_{\eta}$ and $\sigma:=f_{*} \varphi+\eta$.

We will prove the following equation:

$$
\tilde{\nabla}_{Z} \sigma=\langle Z, \varphi\rangle \sigma, \forall Z \in E_{\eta}^{\perp}
$$


By Lemmas 9 and 15, we have that

$$
\left\langle\nabla_{Z}^{\perp} \eta, \xi\right\rangle=-\langle\alpha(Z, \varphi), \xi\rangle \quad \text { and } \quad \nabla_{Z}^{h} \varphi=A_{\eta} Z+\langle Z, \varphi\rangle \varphi,
$$

for all $Z \in E_{\eta}^{\perp}$ and all $\xi \perp \eta$.

By (3.6), $\left(\|\eta\|^{2} \mathrm{Id}-A_{\eta}\right) \varphi=\frac{1}{2} \nabla\|\eta\|^{2}$, thus

$$
\|\eta\|^{2}\langle\varphi, Z\rangle-\left\langle A_{\eta} Z, \varphi\right\rangle=\frac{1}{2} Z\left(\|\eta\|^{2}\right), \forall Z \in E_{\eta}^{\perp} .
$$

In this way, using that $E_{\eta}^{\perp}$ is totally geodesic, we can compute

$$
\tilde{\nabla}_{Z} \sigma=\langle Z, \varphi\rangle f_{*} \varphi+\alpha(\varphi, Z)+\nabla_{Z}^{\perp} \eta
$$

Thus,

$$
\left\langle\tilde{\nabla}_{Z} \sigma, \xi\right\rangle=\langle\alpha(\varphi, Z), \xi\rangle+\left\langle\nabla_{Z}^{\perp} \eta, \xi\right\rangle=0, \forall \xi \perp \eta \text { in } T_{f}^{\perp} M .
$$

If $\eta$ is spacelike or timelike (at some point), then

$$
\begin{aligned}
& \tilde{\nabla}_{Z} \sigma=\langle Z, \varphi\rangle f_{*} \varphi+\left\langle\alpha(Z, \varphi)+\nabla_{Z}^{\perp} \eta, \eta\right\rangle \frac{\eta}{\|\eta\|^{2}}= \\
& =\langle Z, \varphi\rangle f_{*} \varphi+\left[\left\langle A_{\eta} Z, \varphi\right\rangle+\frac{1}{2} Z\left(\|\eta\|^{2}\right)\right] \frac{\eta}{\|\eta\|^{2}}= \\
& \stackrel{(3.22)}{=}\langle Z, \varphi\rangle f_{*} \varphi+\|\eta\|^{2}\langle\varphi, Z\rangle \frac{\eta}{\|\eta\|^{2}}=\langle Z, \varphi\rangle\left(f_{*} \varphi+\eta\right)=\langle Z, \varphi\rangle \sigma .
\end{aligned}
$$

Lets suppose that $\eta$ is lightlike at $x \in M$. In this case, there exists a lightlike vector $\zeta \in T_{x}^{\perp} M$ such that $\langle\eta(x), \zeta\rangle=1$. Thus, at $x$, the following equations hold:

$$
\begin{aligned}
& \tilde{\nabla}_{Z} \sigma=\langle Z, \varphi\rangle f_{*} \varphi+\left\langle\alpha(\varphi, Z)+\nabla_{Z}^{\perp} \eta, \zeta\right\rangle \eta= \\
& =\langle Z, \varphi\rangle f_{*} \varphi+\left[\left\langle A_{\zeta} \varphi, Z\right\rangle-\left\langle\eta, \nabla_{Z}^{\perp} \zeta\right\rangle\right] \eta= \\
& \stackrel{(3.8)}{=}\langle Z, \varphi\rangle\left[f_{*} \varphi+\eta\right]=\langle\varphi, Z\rangle \sigma .
\end{aligned}
$$

Therefore equation (3.21) holds.

Affirmation 1: $\tilde{\nabla}_{Z} f_{*} X=f_{*} \nabla_{Z}^{v} X$, for all $X \in E_{\eta}$ and all $Z \in E_{\eta}^{\perp}$.

If $X \in \Gamma\left(E_{\eta}\right)$ and $Z, W \in \Gamma\left(E_{\eta}^{\perp}\right)$, then $\left\langle\nabla_{Z} X, W\right\rangle=-\left\langle X, \nabla_{Z} W\right\rangle=-\left\langle X, \nabla_{Z}^{v} W\right\rangle=0$, since $E_{\eta}^{\perp}$ is totally geodesic. Thus, $\tilde{\nabla}_{Z} f_{*} X=f_{*} \nabla_{Z} X+\underline{\alpha}(Z, X)=f_{*} \nabla_{Z}^{v} X . \checkmark$

Affirmation 2: The distribution $L:=f_{*} E_{\eta} \oplus[\sigma]$ is parallel in $\mathbb{R}_{t}^{n}$ along $M$, that is, $L=f_{*} E_{\eta} \oplus[\sigma]$ is a constant vector subspace of $\mathbb{R}_{t}^{n}$.

Indeed, if $X \in E_{\eta}$ and $f_{*} Y+\beta \sigma \in f_{*} E_{\eta} \oplus[\sigma]$, then, using that $E_{\eta}$ is spherical and after some computations, we obtain

$$
\tilde{\nabla}_{X}\left(f_{*} Y+\beta \sigma\right)=f_{*}\left[\nabla_{X}^{v} Y-\beta\left(\|\varphi\|^{2}+\|\eta\|^{2}\right) X\right]+[\langle X, Y\rangle+X(\beta)] \sigma
$$

By the other side, using (3.21) and Affirmation 1, we get that

$$
\tilde{\nabla}_{Z}\left(f_{*} Y+\beta \sigma\right)=f_{*} \nabla_{Z}^{v} Y+[Z(\beta)+\beta\langle Z, \varphi\rangle] \sigma .
$$


Therefore $L$ is parallel in $\mathbb{R}_{t}^{n}$ along $M . \checkmark$

We know that $L$ is constant and $f_{*} E_{\eta}$ is spacelike, thus $L$ and $\sigma$ are spacelike at all points of $M$, or $L$ and $\sigma$ are timelike at all points of $M$, or $L$ and $\sigma$ are lightlike at all points of $M$.

Case 1: Lets suppose that $\sigma$ is spacelike.

In this case, using item (III.1) of Proposition 11 and Remarks 12, it follows that the leafs of $E_{\eta}$ are $q$-dimensional ellipsoids in $\mathbb{R}_{t}^{n}$ given by the intersection $\mathbb{S}\left(c(x) ; \frac{1}{\|\sigma(x)\|}\right) \cap(c(x)+L)$, where $\|\sigma(x)\|^{2}$ e $c(x)=f(x)+\frac{\sigma(x)}{\|\sigma(x)\|^{2}}$ are constant in each leaf of $E_{\eta}$.

We stand that $c_{*} T M \perp L$. Indeed, $c$ is constant in the leafs of $E_{\eta}$, thus $c_{*} X=0$, for all $X \in E_{\eta}$. If $Z \in E_{\eta}^{\perp}$, then, using (3.21), we get that

$$
c_{*} Z=f_{*} Z-\frac{\langle Z, \varphi\rangle}{\|\sigma\|^{2}} \sigma .
$$

Thus, $\left\langle c_{*} Z, f_{*} X\right\rangle=0$ and $\left\langle c_{*} Z, \sigma\right\rangle=\left\langle f_{*} Z, \sigma\right\rangle-\langle Z, \varphi\rangle=\langle Z, \varphi\rangle-\langle Z, \varphi\rangle=0$. Therefore $c_{*} T M \perp L$.

Lets consider the manifold $N^{m-q}:=M / \sim$, where $\sim$ is the equivalence relation given by

$$
x \sim y \equiv x \text { and } y \text { are at the same leaf of distribution } E_{\eta} .
$$

We know that $c(x)=f(x)+\frac{\sigma(x)}{\|\sigma\|^{2}}$ and $\|\sigma(x)\|^{2}$ are constant in each leaf of $E_{\eta}$, thus we can define the applications $\bar{c}: N \rightarrow \mathbb{R}_{t}^{n}$ and $r: N \rightarrow \mathbb{R}$ by $\bar{c}(\bar{x}):=c(x)$ e $r(\bar{x}):=\frac{1}{\|\sigma(x)\|}$, where $\bar{x}$ is the equivalence class of $x$.

Let $\Pi: \mathbb{R}_{t}^{n} \rightarrow L$ be the orthogonal projection. Thus, $\Pi \circ c$ and $\Pi \circ \bar{c}$ are constant in $M$ and $N$ respectively, cause $c_{*} T M \perp L$. In this way,

$$
f(x)=c(x)-\frac{\sigma(x)}{\|\sigma\|^{2}}=p+h(\bar{x})-r(\bar{x}) \frac{\sigma(x)}{\|\sigma(x)\|},
$$

where $p:=\Pi(c(x))$ and $h(\bar{x})$ is the orthogonal projection of $\bar{c}(\bar{x})$ on $L^{\perp}$.

Therefore $f(M)$ is an open subset of the rotational submanifold with axis $L^{\perp}$ on the immersion $\bar{f}: N \rightarrow L^{\perp} \ominus \operatorname{span}\{\xi\}$, where $\bar{f}(\bar{x}):=\bar{h}(\bar{x})+\bar{r}(\bar{x}) \xi$ and $\xi \in \mathbb{S}(0,1) \subset L$ is a fixed vector. It's rotational parametrization $g: N \times \mathbb{S}(0,1) \rightarrow \mathbb{R}_{t}^{n}$ is given by $g(\bar{x}, y):=p+h(\bar{x})+r(\bar{x}) y$.

Case 2: Lets suppose that $\sigma$ is timelike.

This case is analogous to the first case. We can prove that $f(M)$ is an open subset of the rotational submanifold with axis $L^{\perp}$ on the immersion $\bar{f}: N \rightarrow L^{\perp} \oplus \operatorname{span}\{\xi\}$, where $\bar{f}(\bar{x}):=\bar{h}(\bar{x})+$ $\bar{r}(\bar{x}) \xi, \xi \in \mathbb{S}(0,-1) \subset L$ is a fixed vector, $N:=M / \sim$ and $\sim$ is the equivalence relation given at Case 1. The rotational parametrization is $g: N \times \mathbb{S}(0,-1) \rightarrow \mathbb{R}_{t}^{n}$, given by $g(\bar{x}, y):=p+h(\bar{x})+r(\bar{x}) y$, $\mathbb{S}(0,-1) \subset L$.

Case 3: Lets suppose that $\sigma$ is lightlike.

In this case, $L=E_{\eta} \ominus \operatorname{span}\{\sigma\}$ is a lightlike subspace subspace of $\mathbb{R}_{t}^{n}$.

Affirmation 4: If $x_{0} \in M$ and $\sigma_{0}=\sigma\left(x_{0}\right)$, then $\sigma(x)=\frac{1}{r(x)} \sigma_{0}$, for some differentiable function $r: M \rightarrow \mathbb{R}$.

If $x_{0} \in M$ and $\left\{X_{1}, \cdots, X_{q}\right\}$ is an orthonormal basis of $E_{\eta}\left(x_{0}\right)$, then $L=\operatorname{span}\left\{X_{1}, \cdots, X_{q}, \sigma\left(x_{0}\right)\right\}$, cause $L$ is constant. Thus, $\sigma(x)=a_{1}(x) X_{1}+\cdots+a_{m}(x) X_{m}+\frac{1}{r(x)} \sigma_{0}$ and $0=\|\sigma(x)\|^{2}=\sum_{i=1}^{m} a_{i}^{2}(x)$. It follows that $a_{1}(x)=\cdots=a_{m}(x)=0$ and $\sigma(x)=\frac{1}{r(x)} \sigma_{0}$. 
Let $V \subset L$ be a spacelike vector subspace and $\tilde{\sigma}_{0}$ be a lightlike vector such that $\tilde{\sigma}_{0} \perp V$ and $\left\langle\sigma_{0}, \tilde{\sigma}_{0}\right\rangle=1$. Thus, $\frac{1}{r(x)}=\left\langle\sigma(x), \tilde{\sigma}_{0}\right\rangle$ is differentiable. $\checkmark$

Lets define $\tilde{\sigma}(x):=r(x) \tilde{\sigma}_{0}$. Thus, $\tilde{\sigma}$ is a lightlike differentiable field such that $\tilde{\sigma} \perp V$ and $\langle\sigma, \tilde{\sigma}\rangle=1$. Besides that, $\mathbb{R}_{t}^{n}=\operatorname{span}\{\sigma, \tilde{\sigma}\} \oplus U \oplus V=\operatorname{span}\left\{\sigma_{0}, \tilde{\sigma}_{0}\right\} \oplus U \oplus V$, where $U=(\operatorname{span}\{\sigma, \tilde{\sigma}\} \oplus V)^{\perp}$ is a nondegenerated vector subspace of $L^{\perp} \subset \mathbb{R}_{t}^{n}$.

Lets consider

$$
\xi(x):=-\sum_{i=1}^{q}\left\langle v_{i}(x), \tilde{\sigma}(x)\right\rangle v_{i}(x)+\frac{1}{2} \sum_{i=1}^{q}\left\langle v_{i}(x), \tilde{\sigma}(x)\right\rangle^{2} \sigma(x)+\tilde{\sigma}(x)
$$

where $v_{i}(x)=f_{*} e_{i}(x)$ e $\left\{e_{1}(x), \cdots, e_{q}(x)\right\}$ is an orthonormal basis of $E_{\eta}(x)$. It can be shown that $\xi$ is a lightlike differentiable field such that, $\xi \perp E_{\eta}, \xi \in L \oplus \operatorname{span}\{\tilde{\sigma}\}=L \oplus \operatorname{span}\left\{\tilde{\sigma}_{0}\right\}$ and $\langle\xi, \sigma\rangle=1$ (see the arguments at Lemma 1.2 of [9]).

By item (III.3) of Proposition 11 and by Remarks 12,

$$
f(x) \in p(x)+(-\tilde{\sigma}(x)+L) \cap \mathscr{L}=p(x)-\tilde{\sigma}(x)+\left\{v+\frac{\|v\|^{2}}{2} \sigma(x) \mid v \in V\right\},
$$

where $p(x)=f(x)+\xi(x)$ is constant in each leaf of $E_{\eta}$.

Let $P: \mathbb{R}_{t}^{n} \rightarrow V$ be the orthogonal projection and $v(x)=P(f(x)-p(x))$. Thus, $f(x)-p(x) \in$ $\operatorname{span}\{\tilde{\sigma}, \sigma\} \oplus V$ and

$$
f(x)=p(x)-\tilde{\sigma}(x)+v(x)+\frac{\|v(x)\|^{2}}{2} \sigma(x)=p(x)+r(x)\left(-\tilde{\sigma}_{0}+w(x)+\frac{\|w(x)\|^{2}}{2} \sigma_{0}\right),
$$

where $w(x):=\frac{v(x)}{r(x)}$.

Affirmation 5: $\left\{v_{*} e_{1}, \cdots, v_{*} e_{q}\right\}$ is an orthonormal basis of $V$.

If $X \in \Gamma\left(E_{\eta}\right)$, then, using that $E_{\eta}$ is spherical and $\eta$ is a Dupin normal, we can get that

$$
\tilde{\nabla}_{X} \sigma=-\|\sigma\|^{2} f_{*} X=0 \text {. }
$$

Thus, $\sigma, \tilde{\sigma}$ and $r$ are constant in the leafs of $E_{\eta}$. But $p$ is also constant in the leafs of $E_{\eta}$, therefore $f_{*} e_{i}=v_{*} e_{i}+\left\langle v, v_{*} e_{i}\right\rangle \sigma\left\langle v_{*} e_{i}, v_{*} e_{j}\right\rangle=\left\langle f_{*} e_{i}, f_{*} e_{j}\right\rangle$ and $\left\{v_{*} e_{1}, \cdots, v_{*} e_{q}\right\}$ is an orthonormal basis of V. $\checkmark$

Affirmation 6: $\tilde{\nabla}_{Z} \tilde{\sigma}=-\langle Z, \varphi\rangle \tilde{\sigma}$, for all $Z \in E_{\eta}^{\perp}$.

By (3.21), $\langle Z, \varphi\rangle \sigma=\tilde{\nabla}_{Z} \sigma=\tilde{\nabla}_{Z} \frac{\sigma_{0}}{r}=-\frac{Z(r)}{r^{2}} \sigma_{0}=-\frac{Z(r)}{r} \sigma$. Thus, $\varphi=-\frac{\nabla r}{r}$ and $\nabla r=-r \varphi$.

Therefore, $\tilde{\nabla}_{Z} \tilde{\sigma}=\tilde{\nabla}_{Z} r \tilde{\sigma}_{0}=Z(r) \tilde{\sigma}_{0}=\langle Z, \nabla r\rangle \tilde{\sigma}_{0}=\langle Z,-r \varphi\rangle \tilde{\sigma}_{0}=-\langle Z, \varphi\rangle \tilde{\sigma} . \checkmark$

We know that $V \subset L$ is a fixed subspace, thus $V \oplus \operatorname{span}\left\{\tilde{\sigma}_{0}\right\}=V \oplus \operatorname{span}\{\tilde{\sigma}\}$ is also a constant subspace. If $\Pi:(\operatorname{span}\{\tilde{\sigma}\} \oplus V) \oplus(\operatorname{span}\{\sigma\} \oplus U) \rightarrow \operatorname{span}\{\tilde{\sigma}\} \oplus V$ is the projection, then $\mathrm{d}(\Pi \circ$ $p)(x) X=0$, for any $X \in E_{\eta}$, because $p$ is constant in the leafs of $E_{\eta}$.

If $Z \in E_{\eta}^{\perp}$, then

$$
\mathrm{d}(\Pi \circ p)(x) Z=\Pi\left(\tilde{\nabla}_{Z} p(x)\right)=\Pi\left[\tilde{\nabla}_{Z}(f+\xi)(x)\right] .
$$


But, using Affirmation 2 and after some computations, we get that

$$
\begin{aligned}
\tilde{\nabla}_{Z}(f+\xi)=f_{*} Z-\sum_{i=1}^{q}\left[\left(\left\langle f_{*} \nabla_{Z}^{v} e_{i}, \tilde{\sigma}\right\rangle-\langle Z, \varphi\rangle\left\langle f_{*} e_{i}, \tilde{\sigma}\right\rangle\right)\right. & \left.f_{*} e_{i}+\left\langle f_{*} e_{i}, \tilde{\sigma}\right\rangle f_{*} \nabla_{Z}^{v} e_{i}\right]+ \\
& +\sum_{i=1}^{q}\left\langle f_{*} e_{i}, \tilde{\sigma}\right\rangle\left\langle f_{*} \nabla_{Z}^{v} e_{i}, \tilde{\sigma}\right\rangle \sigma-\langle Z, \varphi\rangle \tilde{\sigma}
\end{aligned}
$$

By the other side, if $X \in \Gamma\left(E_{\eta}\right)$, then $f_{*} X=v_{*} X+\left\langle v, v_{*} X\right\rangle \sigma$ e $\left\langle f_{*} X, \tilde{\sigma}\right\rangle=\left\langle v, v_{*} X\right\rangle$. Thus

$$
\begin{aligned}
\tilde{\nabla}_{Z}(f+\xi)=f_{*} Z-\sum_{i=1}^{q}\left[\left(\left\langle v, v_{*} \nabla_{Z}^{v} e_{i}\right\rangle-\langle Z, \varphi\rangle\left\langle v, v_{*} e_{i}\right\rangle\right) f_{*} e_{i}+\left\langle v, v_{*} e_{i}\right\rangle f_{*} \nabla_{Z}^{v} e_{i}\right]+ & \\
& +\sum_{i=1}^{q}\left\langle v, v_{*} e_{i}\right\rangle\left\langle v, v_{*} \nabla_{Z}^{v} e_{i}\right\rangle \sigma-\langle Z, \varphi\rangle \tilde{\sigma} .
\end{aligned}
$$

Besides that, we can easily compute that

$$
\Pi(\tilde{\sigma})=\tilde{\sigma} ; \quad \Pi(\sigma)=0 ; \quad \Pi\left(f_{*} X\right)=v_{*} X ; \quad \Pi\left(f_{*} Z\right)=-\langle Z, \varphi\rangle v+\langle Z, \varphi\rangle \tilde{\sigma}
$$

Therefore, after some calculations, we conclude that $\Pi\left[\tilde{\nabla}_{Z}(f+\xi)(x)\right]=0$, that is, $q=\Pi(p(x))$ is constant.

Let $N:=M / \sim$, where $\sim$ is the equivalence relation of Case 1 , and $\pi: \mathbb{R}_{t}^{n} \rightarrow \operatorname{span}\{\sigma\} \oplus U$ is given by $\pi:=\mathrm{Id}-\Pi$. Thus,

$f(x)=q+\pi(p(x))-\tilde{\sigma}(x)+v(x)+\frac{\|v(x)\|^{2}}{2} \sigma(x)=q+h(\bar{x})+\bar{r}(\bar{x})\left(-\tilde{\sigma}_{0}+w(x)+\frac{\|w(x)\|^{2}}{2} \sigma_{0}\right)$,

where $h: N \rightarrow \operatorname{span}\left\{\tilde{\sigma}_{0}\right\} \oplus U$ and $r: N \rightarrow \mathbb{R}$ are given by $h(\bar{x})=\pi(q(x))$ and $\bar{r}(\bar{x})=r(x)$.

Therefore, $f(M)$ is an open subset of the rotational submanifold with axis $\operatorname{span}\left\{\sigma_{0}\right\} \oplus U$ on $\bar{f}: N \rightarrow \operatorname{span}\left\{\tilde{\sigma}_{0}, \sigma_{0}\right\} \oplus U$, where $\bar{f}(\bar{x}):=h(\bar{x})-\bar{r}(\bar{x}) \tilde{\sigma}_{0}$. The rotational parametrization $g: N \times$ $V \rightarrow \mathbb{R}_{t}^{n}$ is given by

$$
g(\bar{x}, w):=q+h(\bar{x})+\bar{r}(\bar{x})\left(-\tilde{\sigma}_{0}+w+\frac{\|w\|^{2}}{2} \sigma_{0}\right) \cdot \bullet
$$

\section{References}

[1] M. do Carmo and M. Dajczer, Rotation hypersurfaces in spaces of constant curvature, Transactions of the American Mathematical Society 277 (1983)

[2] B. Mendonça and R. Tojeiro, Umbilical Submanifolds of $\mathbb{S}^{n} \times \mathbb{R}$, Canadian Journal of Mathematics (2013)

[3] G. Ganchev and V. Milousheva, Quasi-Minimal Rotational Surfaces in Pseudo-Euclidean Four-Dimensinal Space, arXiv:1210.2741 [math.DG]

[4] B.-Y. Chen, Pseudo-Riemannian Geometry, $\delta$-invariants and Applications, World Scientific Publishing, 2011 
[5] M. Dajczer, L. A. Florit and R. Tojeiro, On a class of submanifolds carrying an extrinsic totally umbilical foliation, Israel Journal of Mathematics 125 (2001)

[6] F. Dillen, J. Fastenakels and J. Van der Veken, Rotation hypersurfaces in $\mathbb{S}^{n} \times \mathbb{R}$ and $\mathbb{H}^{n} \times \mathbb{R}$, Note di Matematicay 29 (2009)

[7] Z. Guo and L. Lin, Generalized Rotation Submanifolds in a Space Form, Results in Mathematics 52 (2008)

[8] S. Nölker, Isometric immersions of warped products, Differential Geometry and its Applications 6 (1996)

[9] B. Mendonça, Imersões Isométricas em Produtos de duas Formas Espaciais, Phd. thesis, Universidade Federal de São Carlos, 2012 